Research Article

\title{
Properties of Neutron Noise Induced by Localized Perturbations in an SFR
}

\author{
Hoai-Nam Tran \\ Institute of Research and Development, Duy Tan University, K7/25 Quang Trung, Da Nang 550000, Vietnam
}

Correspondence should be addressed to Hoai-Nam Tran; tranhoainam4@dtu.edu.vn

Received 31 March 2015; Revised 6 June 2015; Accepted 10 June 2015

Academic Editor: Keith E. Holbert

Copyright (C) 2015 Hoai-Nam Tran. This is an open access article distributed under the Creative Commons Attribution License, which permits unrestricted use, distribution, and reproduction in any medium, provided the original work is properly cited.

Investigation of the properties of neutron noise induced by localized perturbations in a sodium-cooled fast reactor has been performed using a multigroup neutron noise simulator. Three representations of the noise source associated with the perturbations of absorption, fission, and scattering cross sections, respectively, were assumed to be located at the first fuel ring around the central assembly. The energy- and space-dependent noise, that is, the amplitude and the phase, was calculated in a wide range of frequencies, for example, $0.1-100 \mathrm{~Hz}$. The results show that in the important energy range (>1.0 keV) where the noise amplitude is significant the phase is almost constant with energy at the calculated frequencies despite the source types. At low frequencies, the variation of the phase is negligibly small at a large distance from the source. The perturbation in several fast groups has a significant contribution and dominates the amplitude and the phase of the induced noise.

\section{Introduction}

Online diagnostics for monitoring the operating status of light water reactors (LWRs) based on analyzing the detector signals of neutron noise were deployed widely in various countries [1-5]. Measurement of the neutron noise in fast reactors, one of the next generation nuclear systems, and a test facility has also been conducted $[3,6,7]$. However, the knowledge and experience of the neutron noise in fast reactors in both measurement and simulation are very little compared to that of LWRs. Most neutron detectors used in LWRs, such as ionization chamber and fission chamber, are sensitive to thermal neutron. In many cases for simplicity one can investigate the neutron noise in the thermal group to represent the behaviour of the detector noise. Thus, recent numerical development for simulating the neutron noise in LWRs is based on two-group diffusion theory [8-11]. Nevertheless, numerical simulation still remains a challenge to reproduce and interpret the measurement data for improving core surveillance. It is a more difficult task for a fast reactor with less knowledge and experience.
Similar to static calculations in deterministic method, the neutron noise in a fast reactor should be calculated based on a multigroup model. In measurement, the detector signal is a combination of the energy-dependent noise with the cross section of the detector as a weighting function. In a sodiumcooled fast reactor (SFR), a fission chamber consisting of fissionable material, for example, ${ }^{235} \mathrm{U}$ or ${ }^{242} \mathrm{Pu}$, coated on the inner wall of the chamber has a major potential for incore fast neutron detection [12]. Thus, the energy-dependent cross section of the detector is complicated. This is also one of the difficulties for numerical simulation in predicting or interpreting the measurement phenomena. To simulate the neutron noise in fast reactors with hexagonal fuel assemblies, a neutron noise simulator was developed based on multigroup diffusion theory $[13,14]$. The tool consists of two modules: a static module for solving the eigenvalue problem of a static state and a noise module for solving the neutron noise equation with a given source in a frequency domain. An application was performed for investigating the neutron noise behaviour induced by periodic core deformation effect in a large SFR [15]. 
In a realistic fluctuation of a system, the noise source can be modelled via a linear combination of the fluctuations of all cross section types in the first order approximation. The contribution of the perturbation of each cross section type in the total source depends on a specific scenario. For instance, the vibration of a control rod in an LWR can be modelled as the vibration of the absorption cross section, while to simulate the noise induced by fuel vibration, it is necessary to consider all cross section types including the fission cross section. The problem in an SFR is even more complicated since the fluctuation of cross sections is strongly energy-dependent and is considered in a multigroup theory. Therefore, prior to assessing realistic scenarios of fluctuations in an SFR, it is worth investigating the properties of the neutron noise induced by the fluctuations of absorption, scattering, and fission cross sections separately. This is because these fluctuations lead to different properties of the noise sources, respectively, and, as a result, different properties of the induced noise.

The present paper aims at investigating the properties of neutron noise induced by localized perturbations in a large SFR core using the noise simulator. Three representations of the noise sources associated with the localized perturbations of absorption, fission, and scattering cross sections, respectively, were assumed to be located at the center of the core. The space- and energy-dependent neutron noise has been calculated in a wide range of frequencies, for example, 0.1$100 \mathrm{~Hz}$.

The paper is organized as follows. Section 2 presents briefly the principles of the neutron noise equation in multigroup diffusion theory which was solved in a frequency domain in the noise simulator. Section 3 describes the core model of a large SFR and the assumption of local perturbations as the noise source. Results and discussion on the properties of the energy- and space-dependent noise at the calculated frequencies are also presented. Finally, some concluding remarks are given in Section 4.

\section{Principles of the Neutron Noise Simulator}

The basis of the neutron noise equation is the assumption of small stationary fluctuations of the system; that is, the averaged value of a time-dependent quantity over time is equal to the static value. Assume that all time-dependent terms $X(\mathbf{r}, t)$ can be split into a stationary component, $X_{0}(\mathbf{r})$, which corresponds to the value at the steady state, plus a small fluctuation, $\delta X(\mathbf{r}, t)$, as

$$
X(\mathbf{r}, t)=X_{0}(\mathbf{r})+\delta X(\mathbf{r}, t)
$$

By assuming the small fluctuations, the first order noise is taken into account, products of fluctuation terms can be neglected from time-dependent diffusion equations, and the result is a linear equation for the fluctuation of the flux. Subtracting the static equation and after performing a Fourier transform of all time-dependent terms, the first order space- and frequency-dependent neutron noise equation in multigroup diffusion theory is written as follows:

$$
\begin{aligned}
-\nabla & \cdot D_{g} \nabla \delta \phi_{g}(\mathbf{r}, \omega)+\Sigma_{t, g}(\omega) \delta \phi_{g}(\mathbf{r}, \omega) \\
= & \frac{1}{k_{\mathrm{eff}}} \chi_{g}(\omega) \sum_{g^{\prime}} \nu \Sigma_{f, g^{\prime}}(\omega) \delta \phi_{g^{\prime}}(\mathbf{r}, \omega) \\
& +\sum_{g^{\prime} \neq g} \Sigma_{s, g^{\prime} \rightarrow g}(\omega) \delta \phi_{g^{\prime}}(\mathbf{r}, \omega)+S_{g}(\mathbf{r}, \omega),
\end{aligned}
$$

where $g$ denotes the energy group, $\delta \phi_{g}$ is the neutron noise in group $g, D_{g}$ is the diffusion coefficient in group $g$, and $\nu \Sigma_{f, g}$ is the production cross section in group $g . \Sigma_{t, g}(\omega)$ is defined as

$$
\Sigma_{t, g}(\omega)=\Sigma_{a, g}(\omega)+\sum_{g^{\prime} \neq g} \Sigma_{s, g} \rightarrow g^{\prime},
$$

with

$$
\Sigma_{a, g}(\omega)=\Sigma_{a, g}+\frac{i \omega}{v_{g}}
$$

where $\Sigma_{a, g}$ is the absorption cross section in group $g . \Sigma_{s, g^{\prime} \rightarrow g}$ is the scattering cross section from group $g^{\prime}$ to group $g, v_{g}$ is the velocity of neutron in group $g$, and $\chi_{g}(\omega)$ is the frequencydependent fission energy spectrum, which is obtained from the equation of delayed neutron as

$$
\chi_{g}(\omega)=\chi_{g}-\sum_{j} \chi_{g, j}^{d} \frac{i \omega \beta}{\lambda_{j}+i \omega}
$$

The last term in (2), $S_{g}(\mathbf{r}, \omega)$, denotes the noise source in group $g$, which is calculated via the fluctuations of the macroscopic cross sections and the static flux, $\phi_{g}$, as follows:

$$
\begin{aligned}
S_{g}(\mathbf{r}, \omega)= & -\delta \Sigma_{a, g}(\omega) \phi_{g}(\mathbf{r})-\sum_{g^{\prime} \neq g} \delta \Sigma_{s, g \rightarrow g^{\prime}} \phi_{g}(\mathbf{r}) \\
& +\sum_{g^{\prime} \neq g} \delta \Sigma_{s, g^{\prime} \rightarrow g}(\omega) \phi_{g^{\prime}}(\mathbf{r}) \\
& +\frac{1}{k_{\mathrm{eff}}} \chi_{g}(\omega) \sum_{g^{\prime}} \delta \nu \Sigma_{f, g^{\prime}}(\omega) \phi_{g^{\prime}}(\mathbf{r}),
\end{aligned}
$$

where $\delta \Sigma_{\alpha}(\omega)$ with $\alpha=\{a, f, s\}$ represents the fluctuation of macroscopic cross sections. In (6), the fluctuation of the diffusion coefficient is neglected. The neutron noise equation (2) is an inhomogeneous equation with an external source, of which all quantities are frequency-dependent, that is, complex quantities. In order to solve the noise equation, it is necessary to define a noise source. Therefore, one needs to know the characteristics of the static state such as the $k_{\text {eff }}$ and 


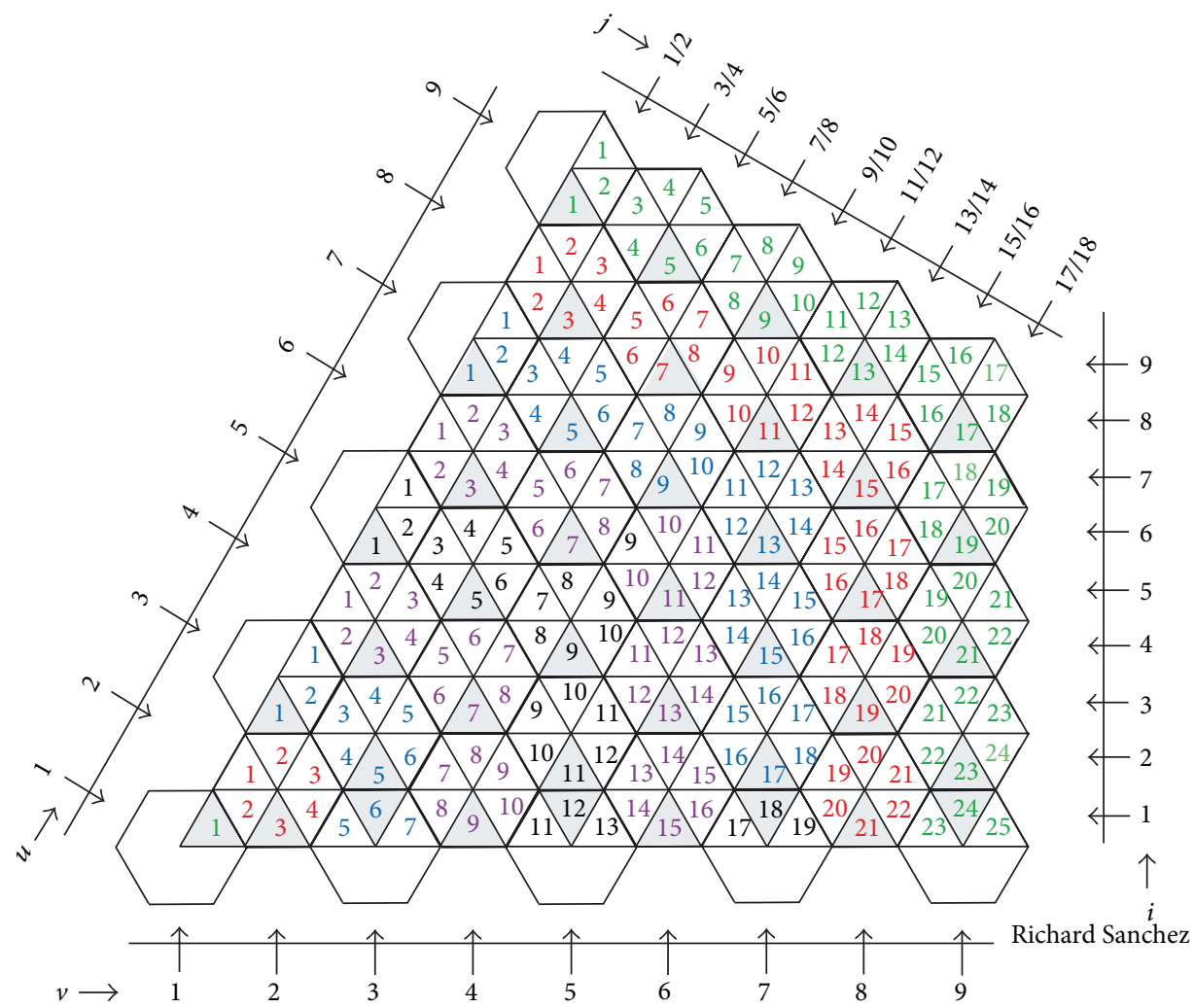

FIGURE 1: Spatial discretization of a hexagonal system in a 60-degree domain.

the static flux, $\phi_{g}(\mathbf{r})$, to calculate the noise source according to (6). This means that the solution of the static equation is also required. The simulator was implemented with two modules: a static module solving the static equation and a noise module solving the noise equation. Finite difference approach is used for the spatial discretization of the system with hexagonal fuel assemblies, where a hexagonal assembly is radially divided into triangular right prisms. Therefore, in a 3D model, each fundamental node has five interfaces including two equilateral triangular bases and three rectangular sides. More detailed description about the noise simulator can be seen in [14].

Figure 1 displays the spatial discretization of a hexagonal system in a 60 -degree domain. In this figure, the $(i / j)$ coordinates are used to handle the triangular fine meshes and the $(u / v)$ coordinates are used to handle the hexagonal coarse meshes. A power iterative solution procedure is implemented for solving the balance equations of both the static and the noise equations. A coarse mesh finite difference (CMFD) method is employed for accelerating the convergence of both the static and the noise solutions, in which a coarse mesh is radially defined as a hexagonal assembly. In previous works, benchmarking calculations for the static state of the ESFR core were performed and had a good agreement with ERANOS [14]. Noise calculations in a two-group model had also a good agreement with analytical solutions [13]. These results give certain assurance for the noise calculations and further investigation of the noise behaviour in fast reactors.

\section{Calculations of the Neutron Noise in an SFR}

3.1. Core Model. In the present work, numerical calculations have been performed based on a $2 \mathrm{D}$ model of European sodium-cooled fast reactor (ESFR) [16] using the multigroup noise simulator [14]. The core is designed with an output power of $3600 \mathrm{MWt}$. The active core consists of two radial fuel regions with a height of $1.0 \mathrm{~m}$ and an equivalent diameter of $4.7 \mathrm{~m}$. The inner fuel region consists of $201 \mathrm{fuel}$ assemblies of mixed U-Pu oxide fuel with $\mathrm{Pu}$ enrichment of $14 \mathrm{wt} \%$. The outer fuel region consists of 252 fuel assemblies of mixed U$\mathrm{Pu}$ oxide with $\mathrm{Pu}$ enrichment of $16 \mathrm{wt} \%$. The radial reflector region consists of 234 steel assemblies. The operation of the core is controlled by 24 control rods and 9 safety rods. The cross section data and the kinetic parameters of a 3D model at the beginning of cycle were taken from previous works which were performed for verifying the tool and for simulating periodic core deformation of the ESFR core $[14,15]$. The data are then processed for a $2 \mathrm{D}$ model in this calculation for the purpose of investigating the properties of the neutron noise on an axial plane. The radial configuration of $1 / 6$ th of the core constructed by 128 assemblies is shown in Figure 2.

3.2. Modelling of the Noise Source. Numerical calculations have been performed based on 33 energy groups and 8 groups of delayed neutron precursors using the neutron noise simulator to investigate the properties of the spaceand frequency-dependent neutron noise. In general, realistic 


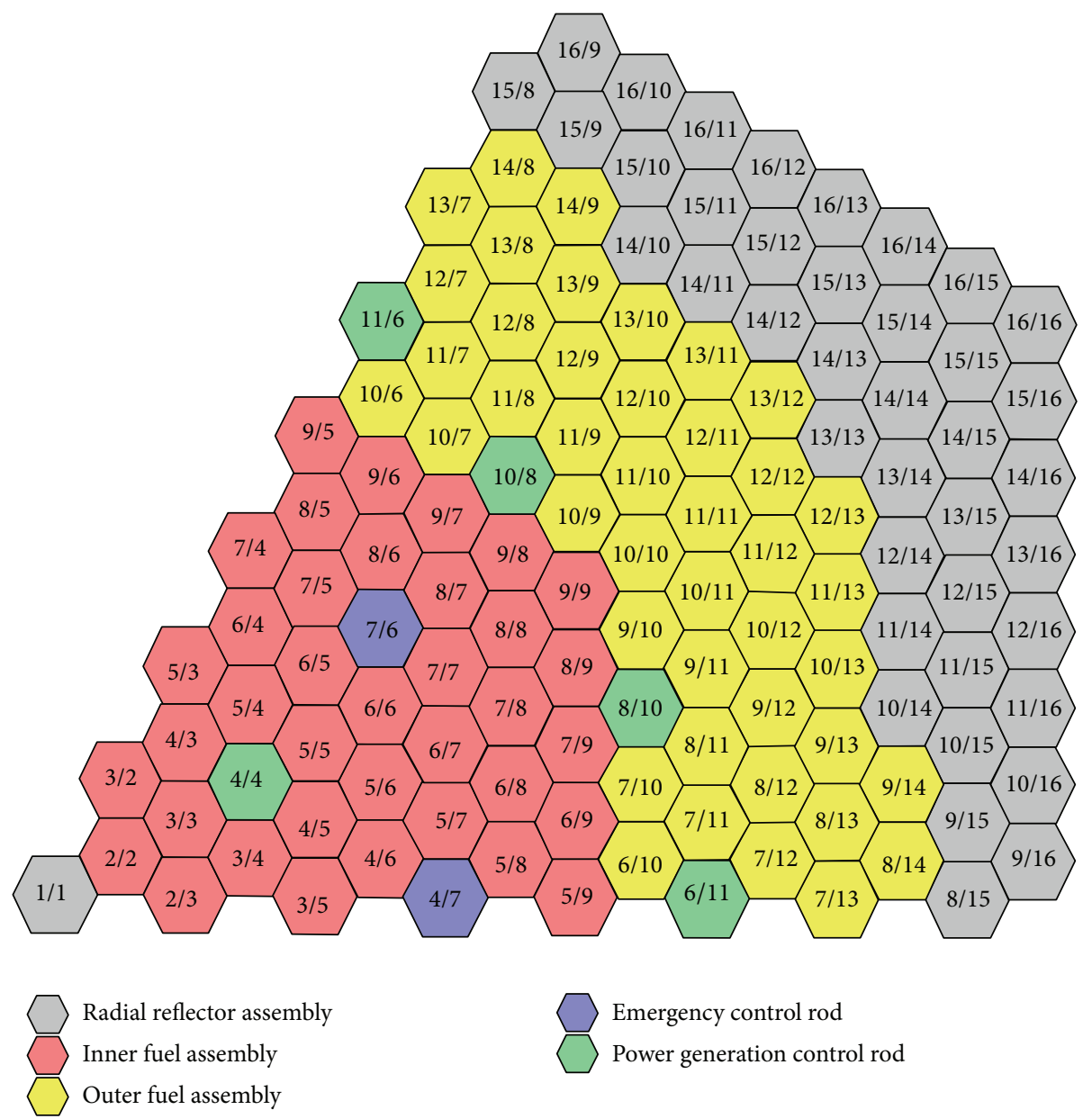

FIGURE 2: Radial configuration of 1/6th of the ESFR core.

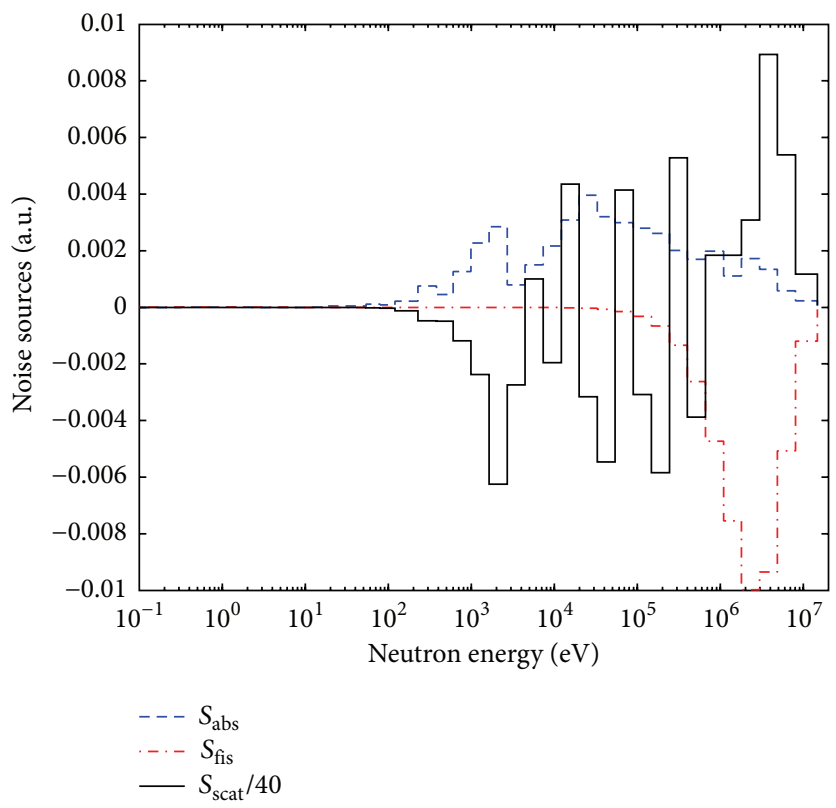

FIGURE 3: Three representations of the noise source associated with the perturbations of absorption, fission, and scattering cross sections denoted as $S_{\text {abs }}, S_{\text {fis }}$, and $S_{\text {scat }}$, respectively, are assumed to be located at the core center. 


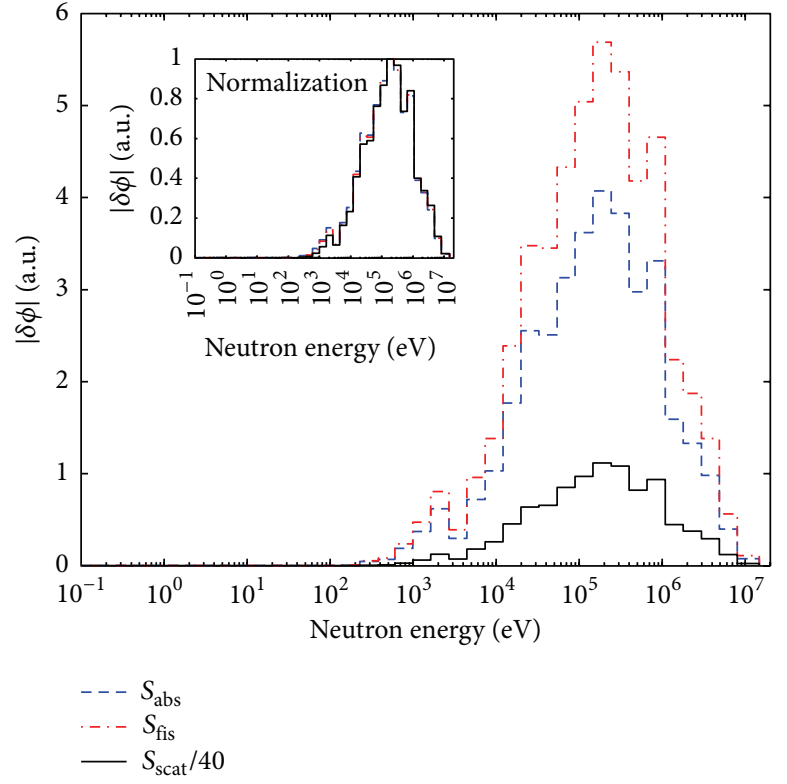

(a)

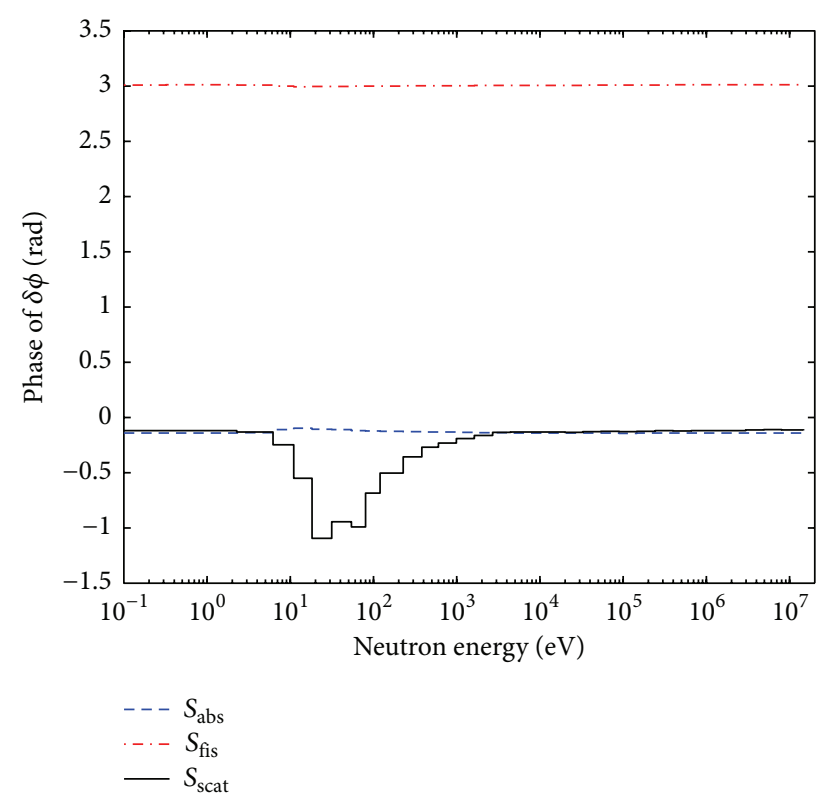

(b)

FIgURE 4: Amplitude (a) and phase (b) of the noise at the location of assembly (3/3) (close to the source) as a function of neutron energy. The noise is calculated at a frequency of $1.0 \mathrm{~Hz}$.

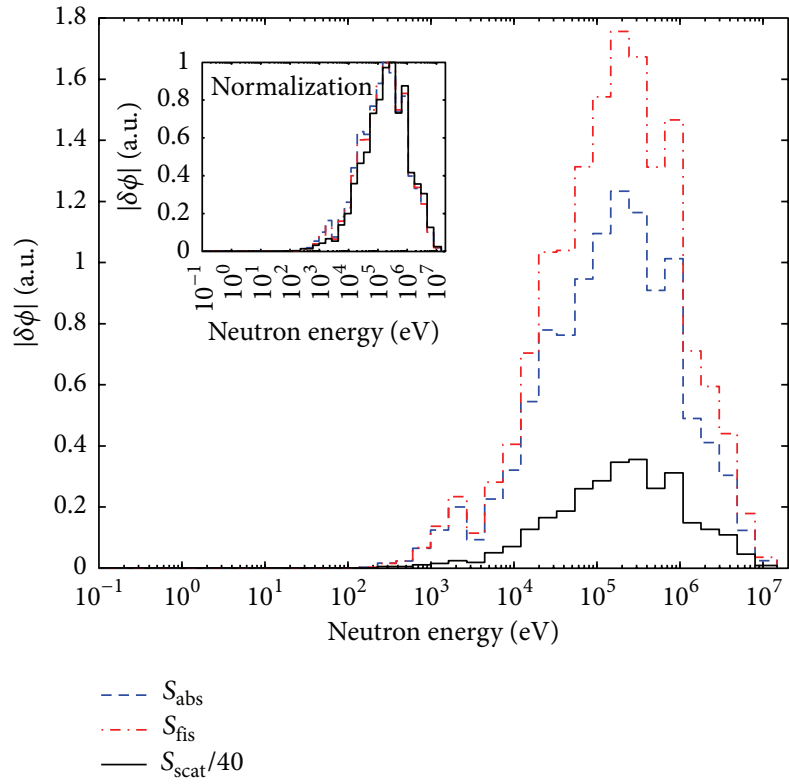

(a)

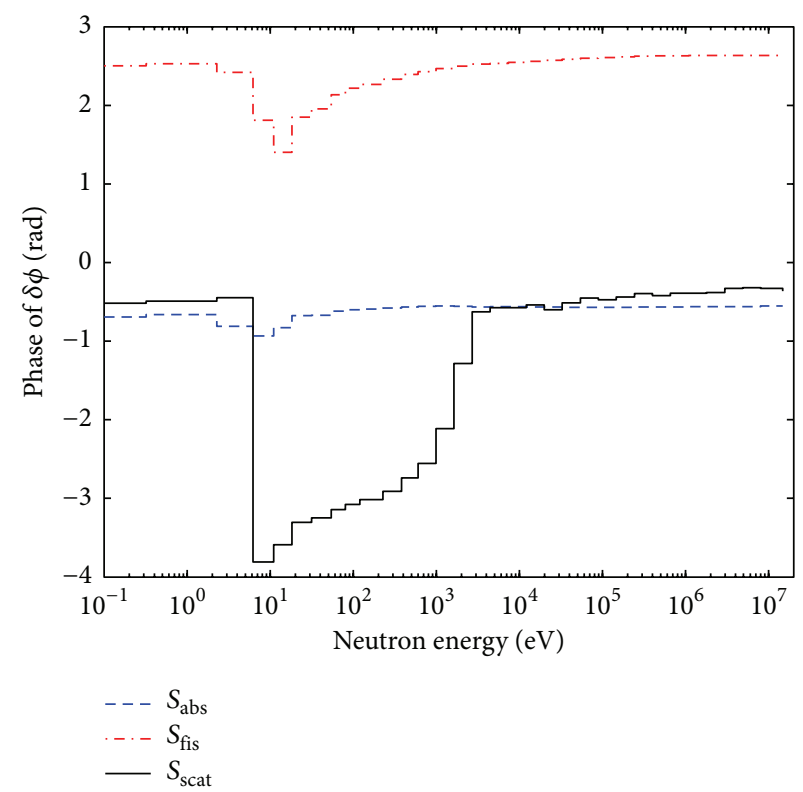

(b)

Figure 5: Amplitude (a) and phase (b) of the noise at the location of assembly (3/3) (close to the source) as a function of neutron energy. The noise is calculated at a frequency of $100 \mathrm{~Hz}$.

fluctuations in a nuclear system can be modelled via the fluctuations of the macroscopic cross sections, and, then, the total noise source, which is a linear combination of all fluctuations, is calculated according to (6) using the static flux and the fluctuations of the cross sections. Therefore, prior to assessing realistic fluctuations, it is worth investigating the properties of the noise induced by the perturbation of each cross section type separately.

Assume that the noise source is originated from the small fluctuation of sodium density in the first fuel ring around the central assembly. This leads to the perturbations of the macroscopic cross sections such as the absorption, 


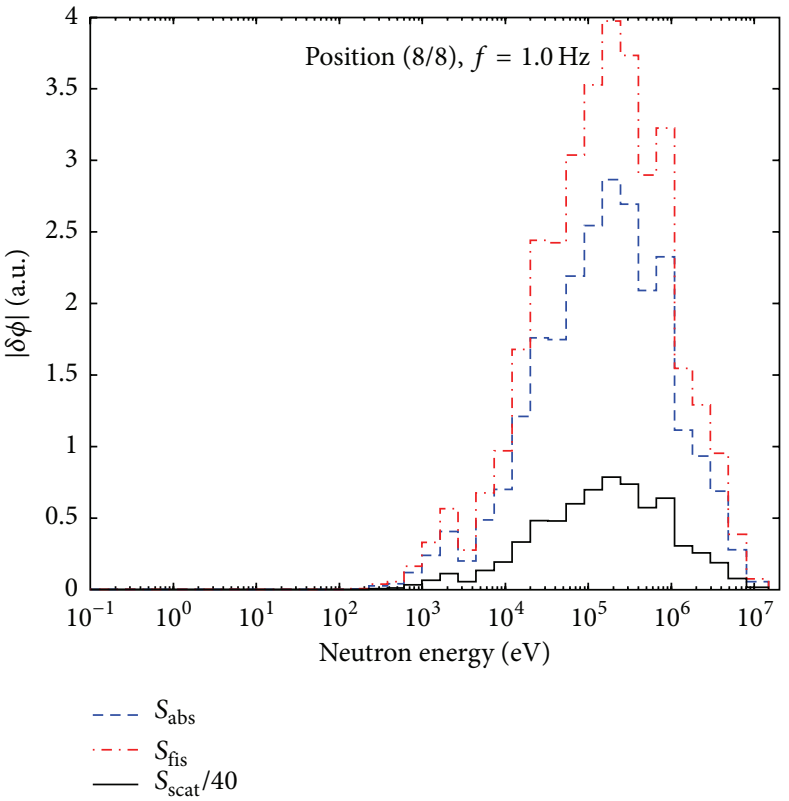

(a)

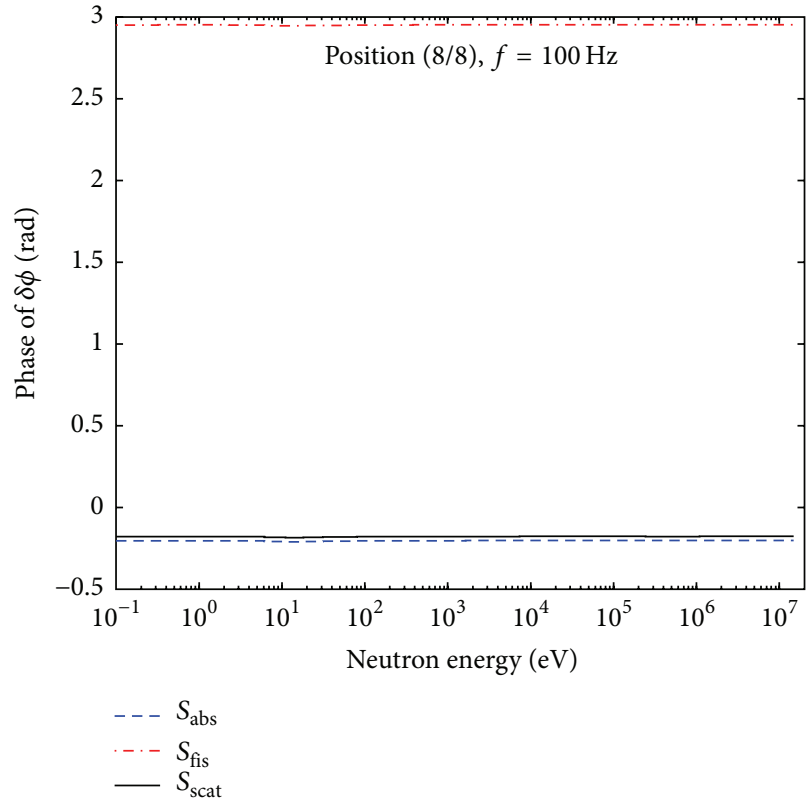

(b)

Figure 6: Amplitude (a) and phase (b) of the noise at the position of assembly (8/8) (far from the source) as a function of neutron energy. The noise is calculated at a frequency of $1.0 \mathrm{~Hz}$.

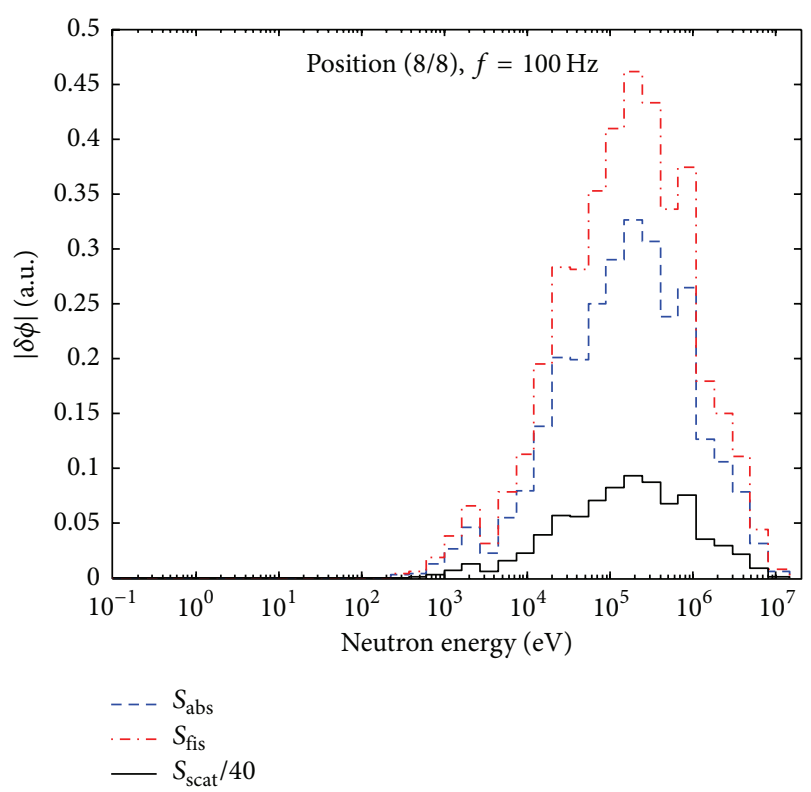

(a)

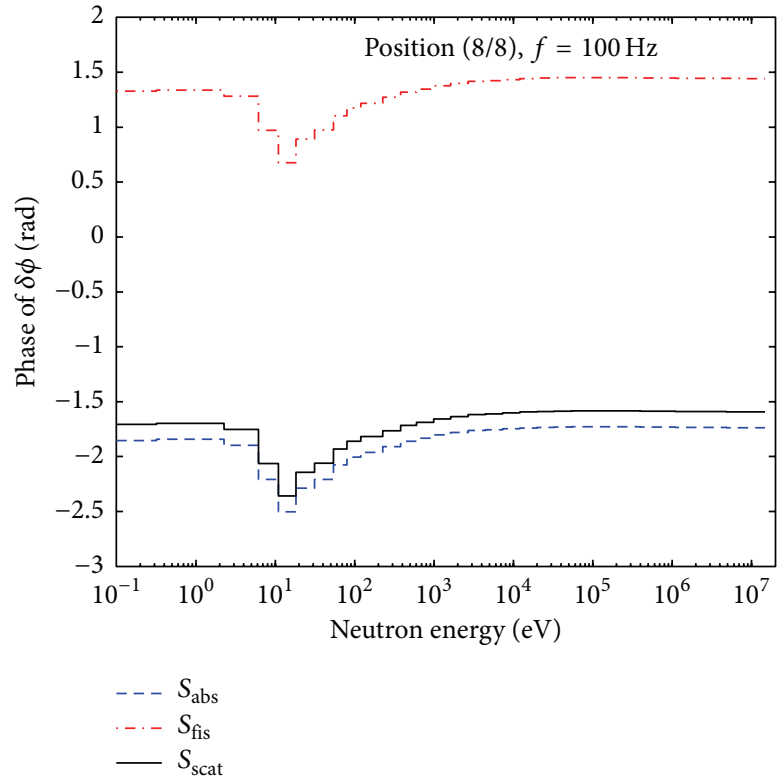

(b)

Figure 7: Amplitude (a) and phase (b) of the noise at the position of assembly (8/8) (far from the source) as a function of neutron energy. The noise is calculated at a frequency of $100 \mathrm{~Hz}$.

fission, and scattering cross sections taking into account the spectrum shift in this area. These perturbations are then represented by three types of the noise sources associated with the perturbations of absorption, fission, and scattering cross sections denoted as $S_{\alpha}, \alpha=\{$ abs, fis, scat $\}$, respectively. With this assumption, the noise sources are calculated using
(6) and displayed in Figure 3. Since the amplitude of the $S_{\text {scat }}$ is greater than that of the $S_{\text {abs }}$ and $S_{\text {fis }}$ by a factor of 40 , it dominates the behaviour of the total induced neutron noise. Therefore, to investigate the total noise it is sufficient to investigate the noise induced by $S_{\text {scat }}$ in this case. However, a general perturbation could be sufficiently represented by 


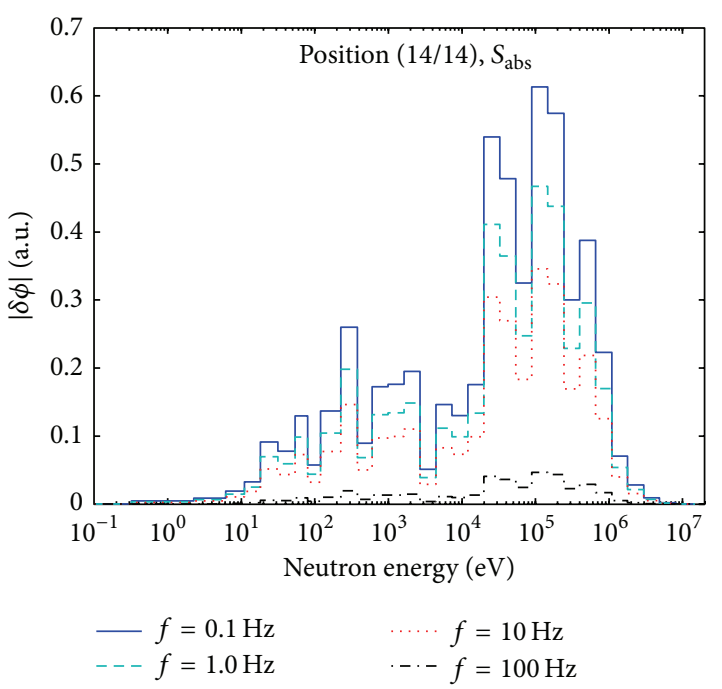

(a)

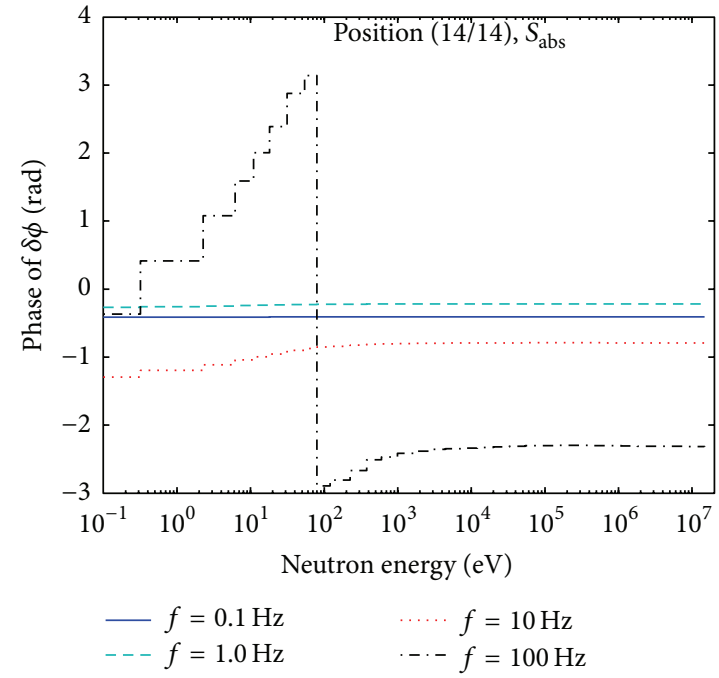

(b)

FIGURE 8: Amplitude (a) and phase (b) of the noise at the position (14/14) in the reflector as a function of neutron energy. The noise is calculated at several frequencies of 0.1 to $100 \mathrm{~Hz}$.

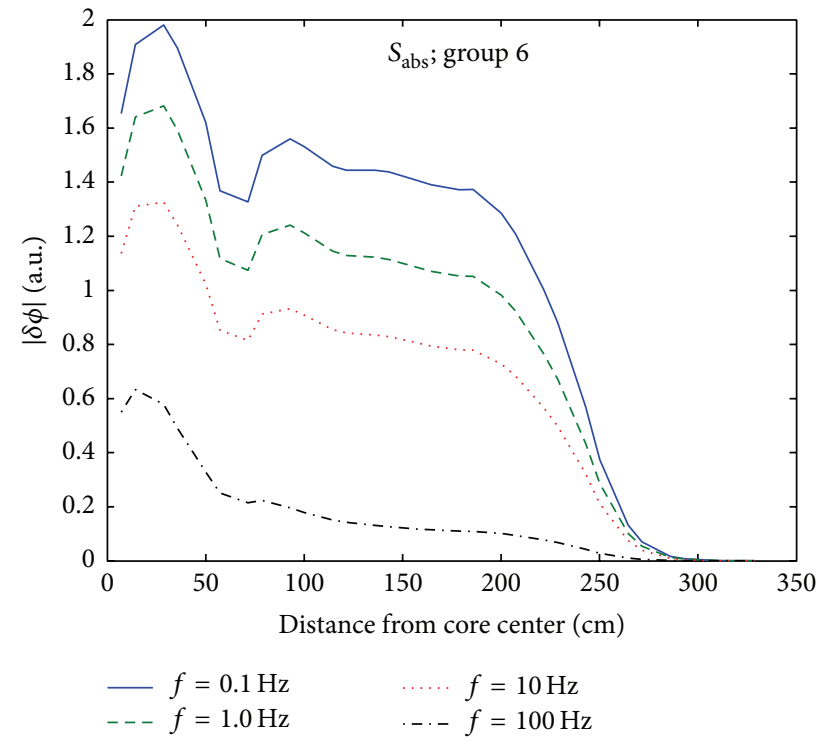

(a)

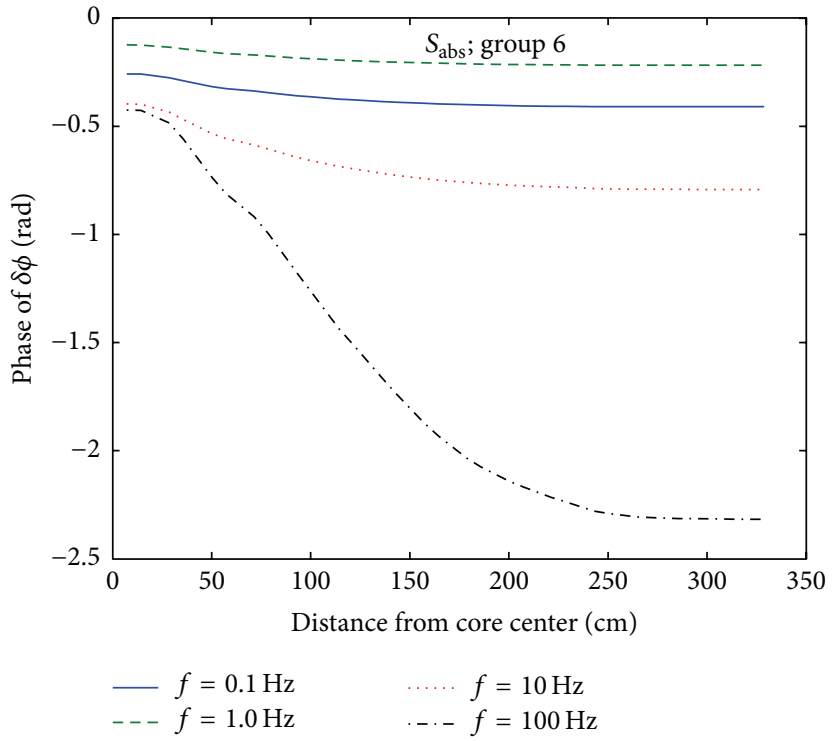

(b)

FIGURE 9: Amplitude (a) and phase (b) of the noise in group 6 along the core radius induced by $S_{\text {abs }}$.

a combination of them. Hence investigation of the behaviour of the noise induced by the three noise sources separately is needed. It can be seen in Figure 3 that the three sources have different properties as a function of neutron energy. The source associated with the perturbation of the fission cross section, $S_{\text {fis }}$, has an opposite phase to that associated with the perturbation of the absorption cross section, $S_{\text {abs }}$, whereas, the source associated with the perturbation of the scattering cross section, $S_{\text {scat }}$, oscillates with energy groups. It is because a downscattering reaction in a certain group leads to a negative source in this group but a positive source in lower energy groups.
The amplitude of the noise sources at energy $<0.1 \mathrm{keV}$ is negligibly small compared to that in higher energy due to the fact that the static flux in this energy range is smaller by three orders of magnitude, or greater. Since most of the neutrons generated by fission are fast neutrons, the source $S_{\text {fis }}$ is negligibly small up to $100 \mathrm{keV}$. The perturbations are assumed to be uniformly distributed in the first fuel ring around the central assembly. Due to the symmetrical property, the noise calculations have been performed in a 60-degree domain, that is, $1 / 6$ th of the core, at several frequencies of $0.1,1.0$, 10 , and $100 \mathrm{~Hz}$ to investigate the properties of the spaceand frequency-dependent noise. In the calculated domain as 


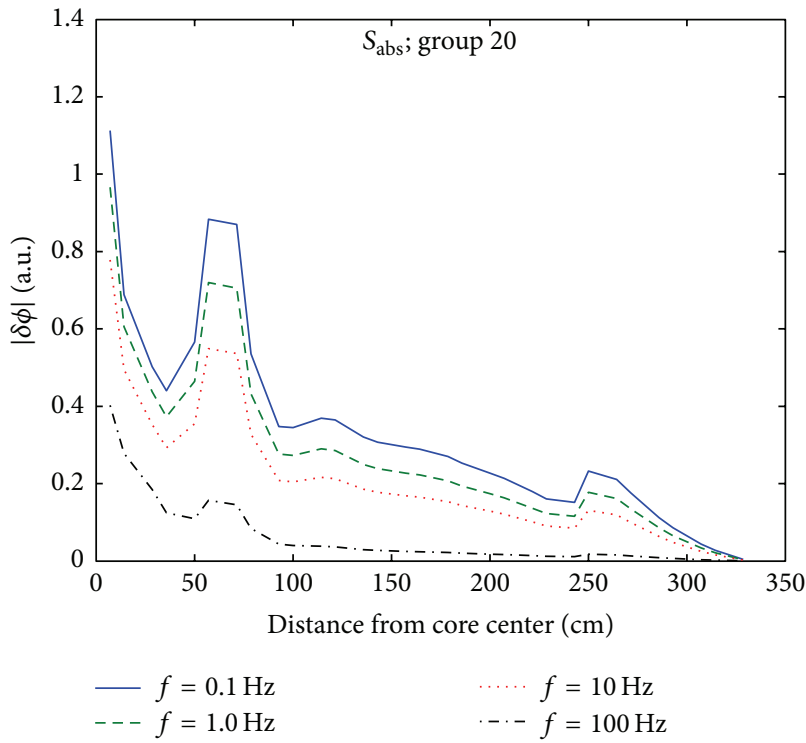

(a)

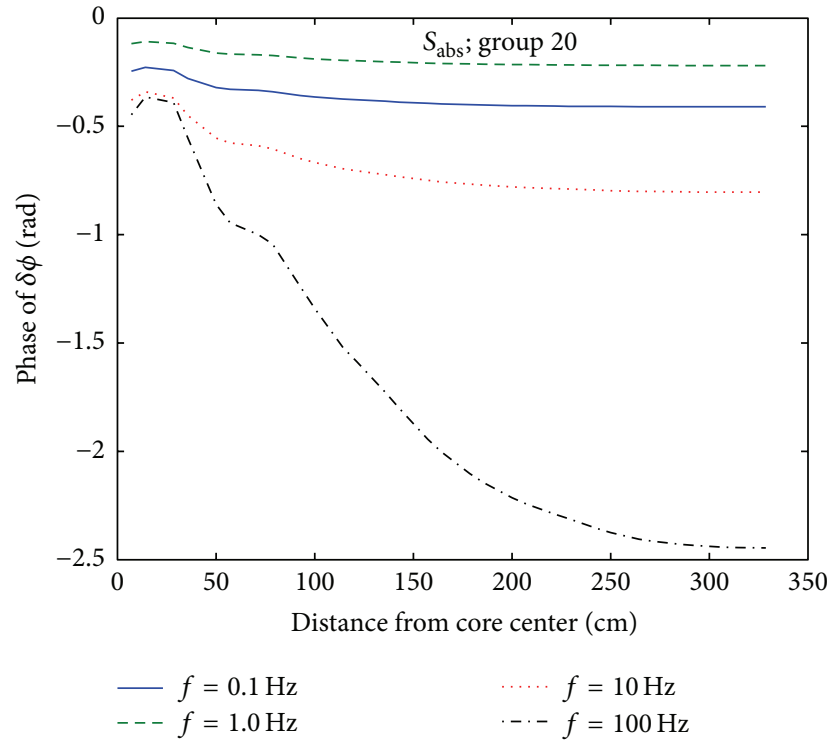

(b)

FIGURE 10: Amplitude (a) and phase (b) of the noise in group 20 along the core radius induced by $S_{\text {abs }}$.

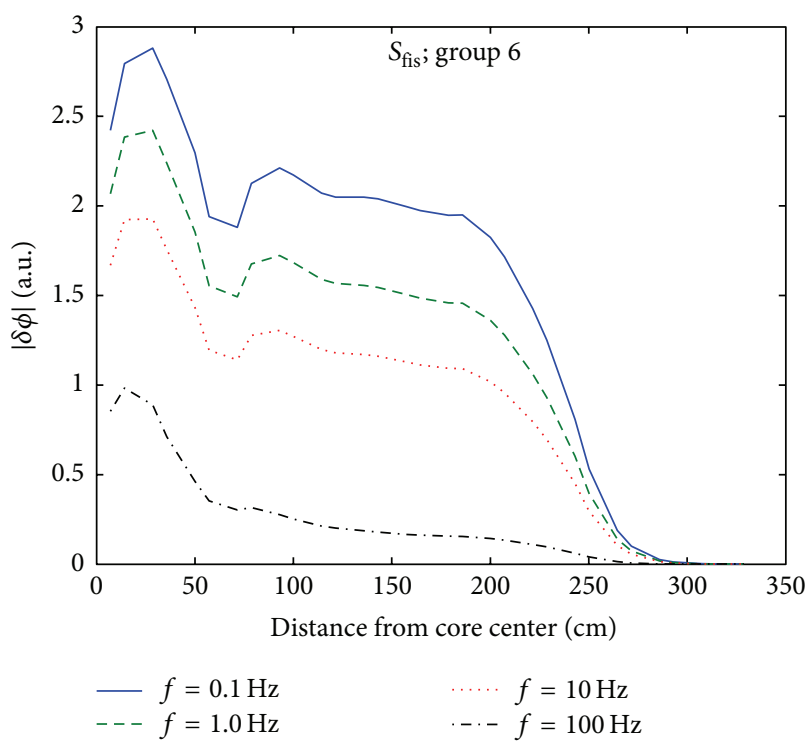

(a)

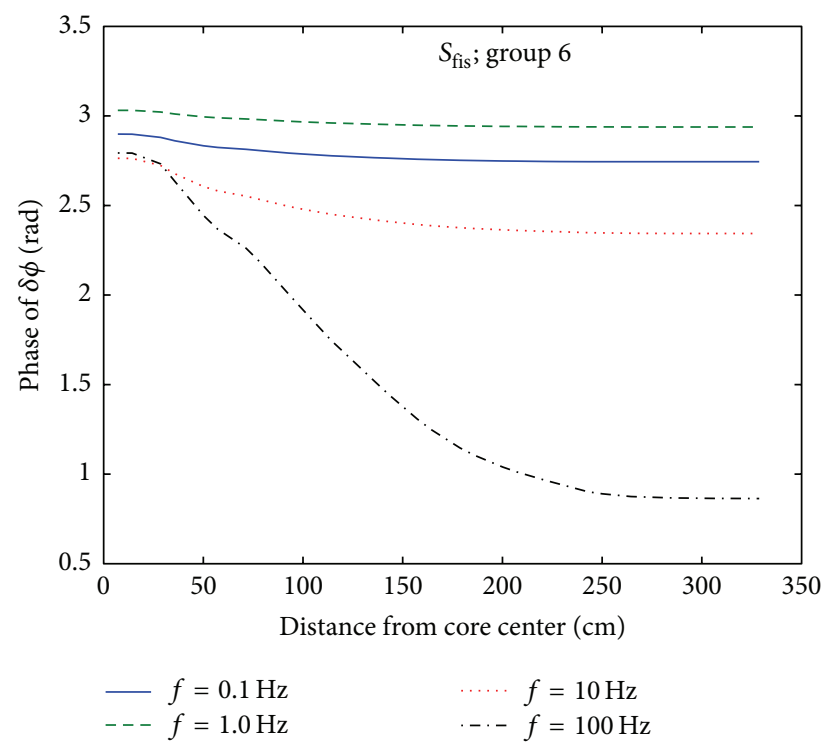

(b)

Figure 11: Amplitude (a) and phase (b) of the noise in group 6 along the core radius induced by $S_{\text {fis }}$.

shown in Figure 2, the perturbation is located in assembly $(2 / 2)$.

\subsection{Neutron Noise Induced by Localized Perturbations}

3.3.1. Energy-Dependent Noise. Numerical calculations have been performed to investigate the noise behaviour in a wide range of frequencies with the noise sources given in Figure 3. The neutron noise can be described as a sum of two components known as a local and a global component. The former is a particular solution of the inhomogeneous equation (2) which dominates the locations close to the source, whereas the latter is related to the point-kinetic response of the reactor. Since the location (3/3) is close to the source, the local component of the noise has more effect. Figure 4 illustrates the amplitude and the phase of the induced neutron noise as a function of energy at a frequency of $1.0 \mathrm{~Hz}$ (low frequency) at the location of assembly (3/3) (close to 


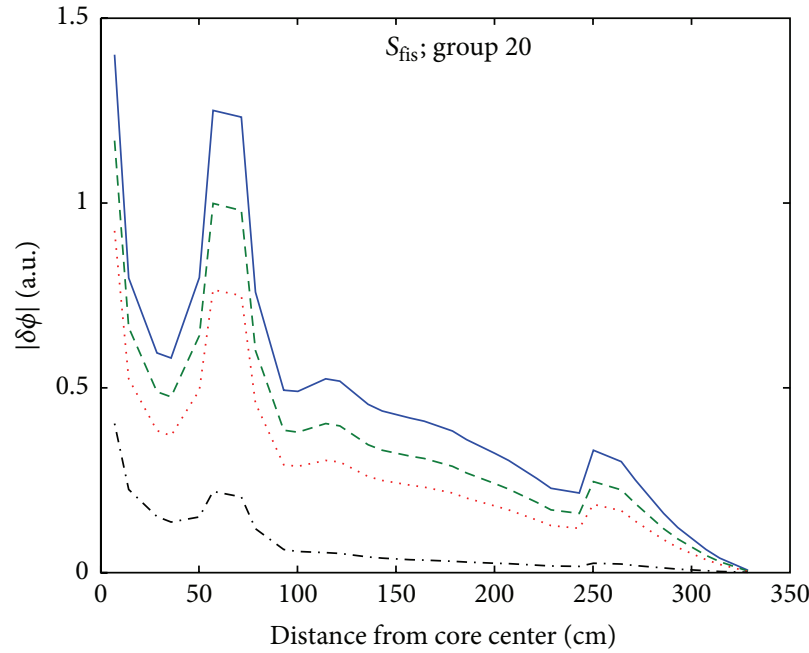

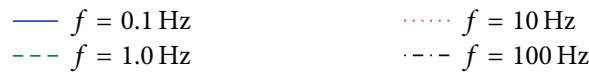

(a)

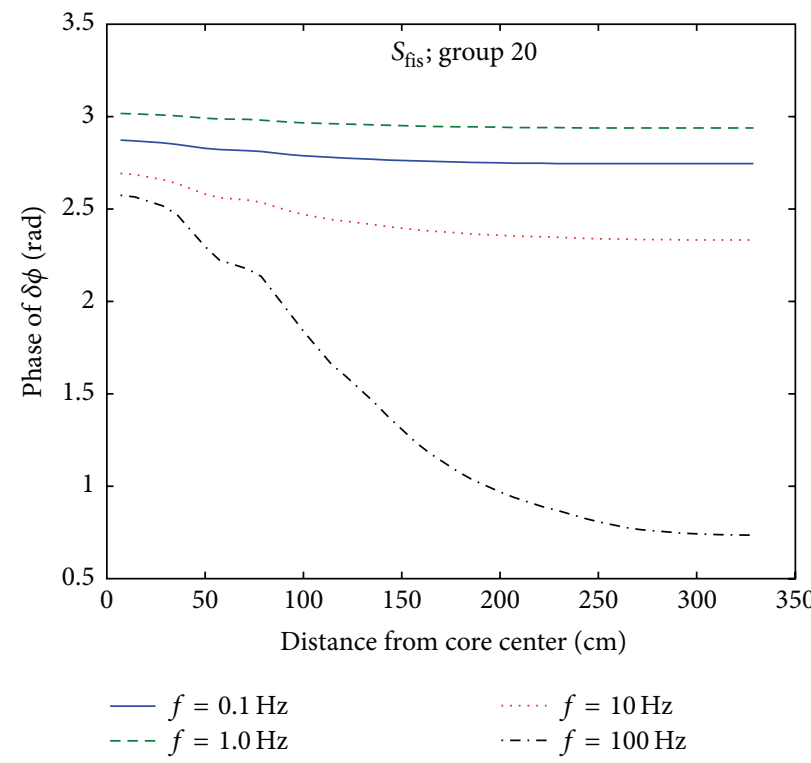

(b)

FIGURE 12: Amplitude (a) and phase (b) of the noise in group 20 along the core radius induced by $S_{\text {fis }}$.

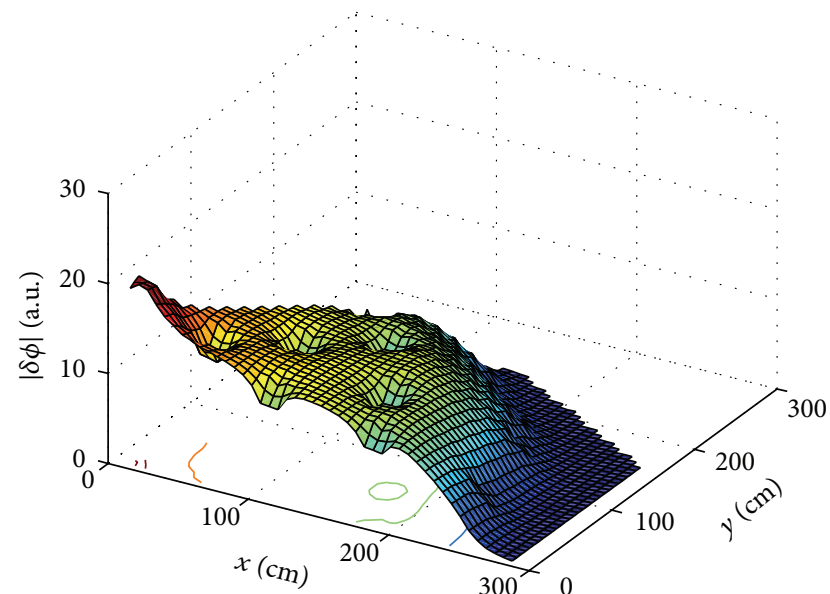

(a)

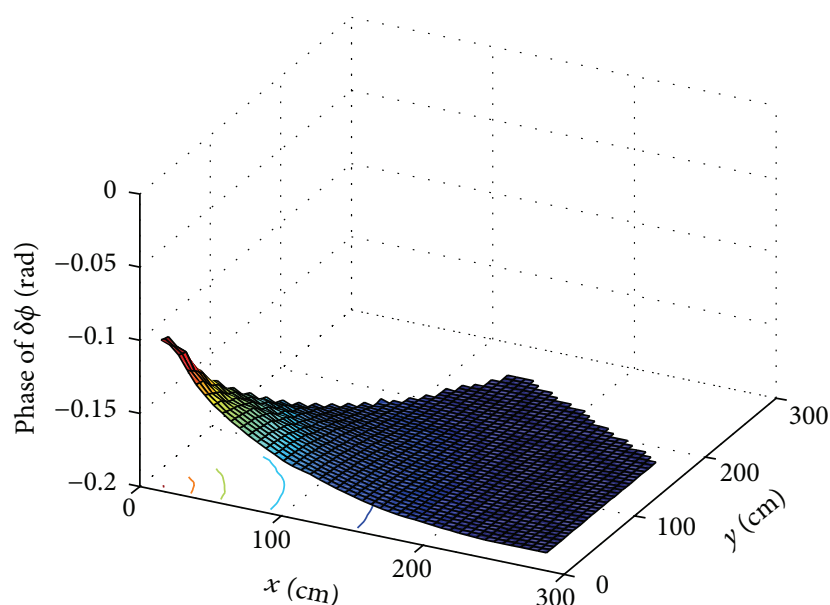

(b)

FIgURE 13: Amplitude (a) and phase (b) of the space-dependent noise in group 6 induced by $S_{\text {scat }}$ at $1.0 \mathrm{~Hz}$.

the source). Figure 5 shows the same quantities as Figure 4 but at a frequency of $100 \mathrm{~Hz}$ (high frequency). Similar to the neutron spectrum, the amplitude of the induced noise is only significant in the energy range $>1.0 \mathrm{keV}$. Comparing among the three cases, at the energy $<100 \mathrm{keV}$ the noise induced by $S_{\text {abs }}$ and $S_{\text {fis }}$ is slightly thermalized compared to that induced by $S_{\text {scat }}$. The out-of-phase behaviour of the $S_{\text {scat }}$ in successive groups in this energy range leads to the cancellation in the induced noise. The normalized amplitude of the noise induced by $S_{\text {abs }}$ at energy $<100 \mathrm{keV}$ is slightly greater than that induced by $S_{\text {fis }}$ despite the significantly greater magnitude of
$S_{\text {abs }}$ in this energy range. This means that the source at energy $>100 \mathrm{keV}$ has more significant contribution to the induced noise over the energy range.

The phase of the noise is almost constant with energy at a frequency of $1.0 \mathrm{~Hz}$, except some variation of the phase in the case of $S_{\text {scat }}$ in the thermal and epithermal energies as shown in Figure 4. At a higher frequency $(100 \mathrm{~Hz})$, the variation of the phase is larger in the thermal and epithermal energy ranges, but in the important range $(>1.0 \mathrm{keV})$ the phase remains constant with energy. The noise induced by $S_{\text {abs }}$ and $S_{\text {fis }}$ is out-of-phase due to the out-of-phase behaviour 


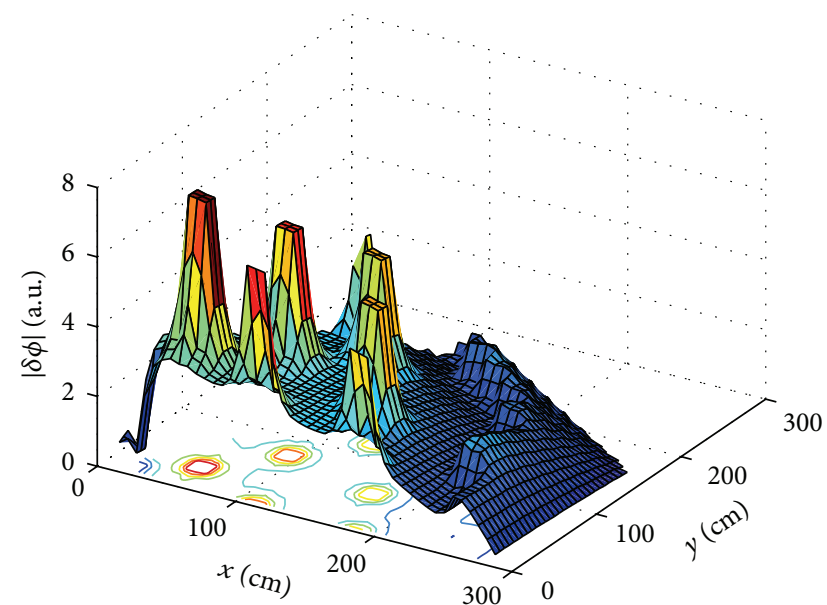

(a)

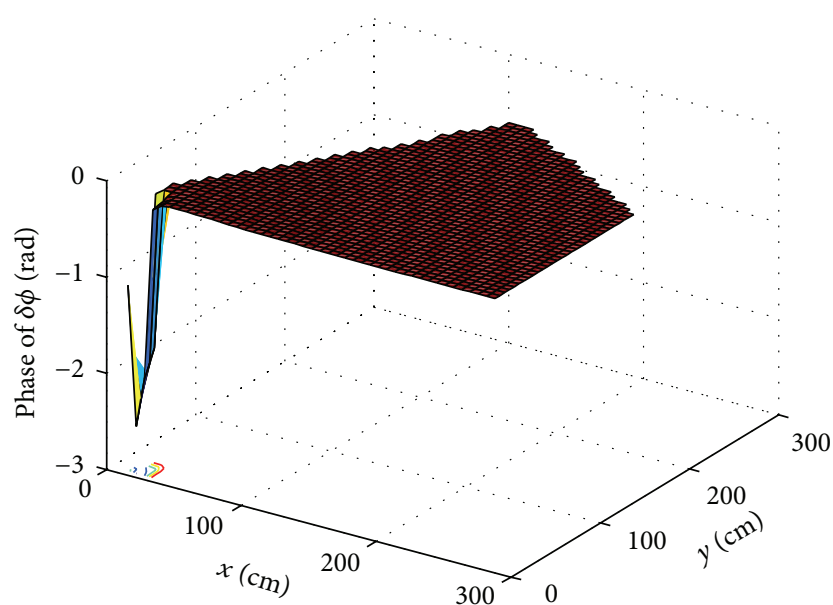

(b)

FIgURE 14: Amplitude (a) and phase (b) of the space-dependent noise in group 20 induced by $S_{\text {scat }}$ at $1.0 \mathrm{~Hz}$.

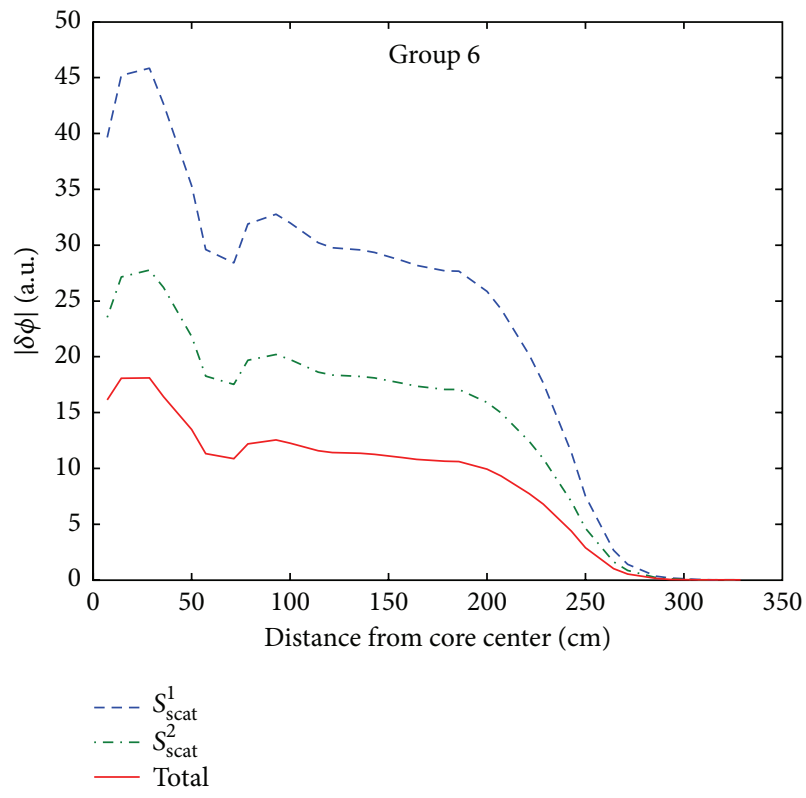

(a)

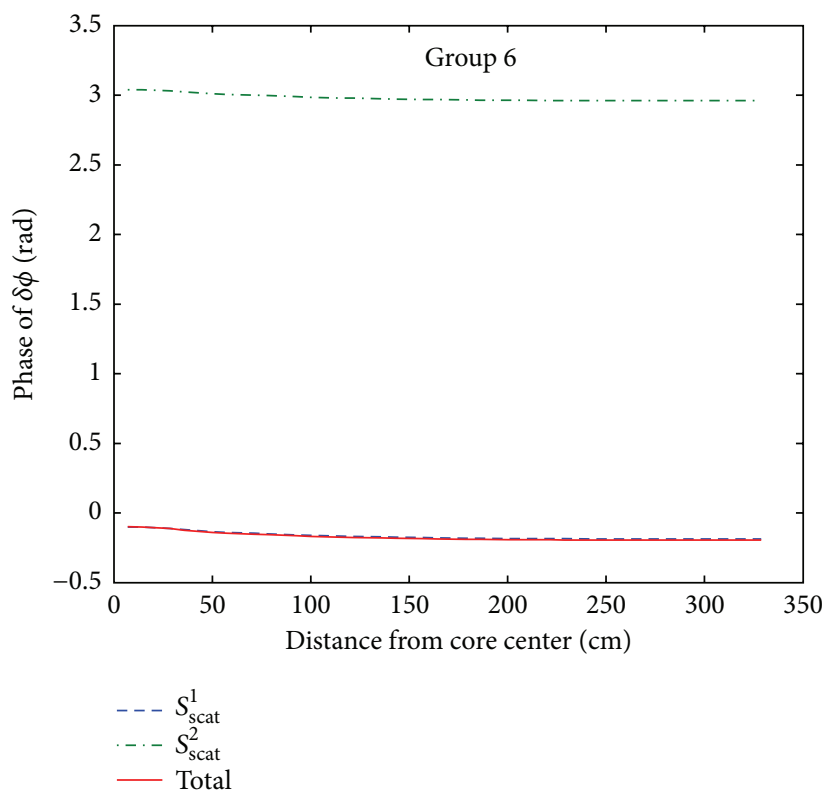

(b)

FIGURE 15: Amplitude (a) and phase (b) of the noise in group 6 along the core radius induced by $S_{\text {scat }}$ at $1.0 \mathrm{~Hz}$. $S_{\text {scat }}$ is assumed to be divided into two terms $S_{\text {scat }}^{1}$ and $S_{\text {scat }}^{2}$ representing the sources in the first six fast groups and in the rest of energy range, respectively.

of the two sources, whereas the phases of the noise induced by $S_{\text {abs }}$ and $S_{\text {scat }}$ are approximately equal in the important energy range $(>1.0 \mathrm{keV})$ despite the oscillation of $S_{\text {scat }}$ with energy.

Figures 6 and 7 show the same quantities as Figures 4 and 5 but at the location of assembly (8/8) in the midcore (far from the source). Figure 8 shows the amplitude and phase of the noise at position $(14 / 14)$ in the reflector as a function of energy at several frequencies. At the positions far from the source, the local component has no effect and the normalized noise amplitudes are identical despite the source types. As shown in Figures $6-8$, the phase at the positions is almost constant with energy at low frequencies. This behaviour is the same with the three sources.

At a high frequency, the variation of the phase in the thermal and epithermal energy ranges increases, but in the important energy range $(>1.0 \mathrm{keV})$ the phase remains constant (see Figures 7 and 8). Principally, the frequency affects the phase of the noise via the ratio $\omega / v$ in (4) and the term related to the delayed neutrons in (5). In the fast energy, that is, high velocity of neutron, $\omega / v$ becomes small and has less effect on the phase of the induced noise even at a high frequency. The phase of the noise induced by $S_{\text {fis }}$ is opposite to 


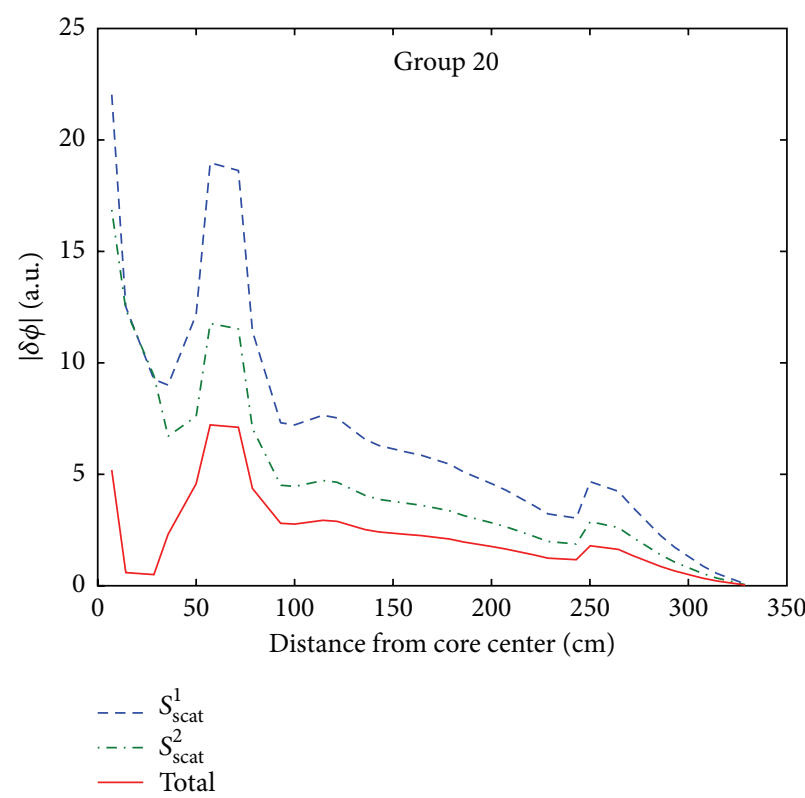

(a)

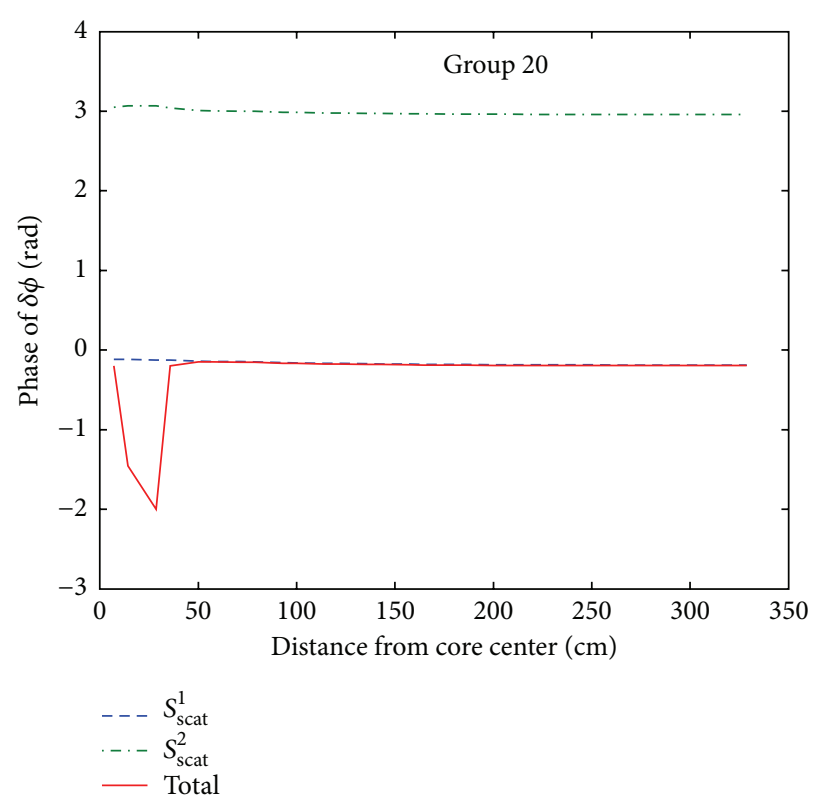

(b)

FIGURE 16: Amplitude (a) and phase (b) of the noise in group 20 along the core radius induced by $S_{\text {scat }}$ at $1.0 \mathrm{~Hz}$. $S_{\text {scat }}$ is assumed to be divided into two terms $S_{\text {scat }}^{1}$ and $S_{\text {scat }}^{2}$ representing the sources in the first six fast groups and in the rest of energy range, respectively.

that induced by $S_{\text {abs }}$ similar to that found near the source due to the out-of-phase behaviour of the two sources. The phase induced by $S_{\text {scat }}$ at a position far from the source is approximately equal to that induced by $S_{\text {abs }}$ over the energy range.

3.3.2. Space-Dependent Noise. Figures 9 and 10 display the amplitude and the phase of the space-dependent noise along the core radius in group 6 (a fast group in the energy range $8.21 \times 10^{5}$ to $1.35 \times 10^{6} \mathrm{eV}$ ) and group 20 (an epithermal group in the energy range $7.49 \times 10^{2}$ to $\left.1.23 \times 10^{3} \mathrm{eV}\right)$ induced by $S_{\text {abs }}$, respectively. Figures 11 and 12 show the same quantities but induced by $S_{\text {fis }}$. One can see that the noise amplitude in the fast group decreases with the increase of the frequency. The local peak of the noise at the position of the source decreases fast with the distance from the source. This behaviour is similar to that found in LWRs. Variation of the phase along the core diameter at frequencies of $0.01-10 \mathrm{~Hz}$ is much smaller compared to that in a frequency of $100 \mathrm{~Hz}$. As illustrated in Figures 9 and 11, the noises induced by $S_{\text {abs }}$ and $S_{\text {fis }}$ have quite similar behaviour but with opposite phases. This indicates that the source in the energy range $<100 \mathrm{keV}$ has less effect than that at higher energy.

Figures 13 and 14 show the amplitude and phase of the space-dependent noise in group 6 (fast group) and group 20 (epithermal group), respectively, induced by $S_{\text {scat }}$ at $1.0 \mathrm{~Hz}$. A local peak can be seen at the source position in the fast group, but in the epithermal group it is a local depth. This is because the out-of-phase behaviour of $S_{\text {scat }}$ in successive energy groups leads to the cancellation in the total noise. The behaviour can be seen more clearly in Figures 15 and 16.

In order to evaluate the contribution of the perturbation at different energy, it is assumed that the source $S_{\text {scat }}$ is divided into two components: one corresponds to the perturbation in the six fast groups (highest energy) denoted as $S_{\text {scat }}^{1}$ and another corresponds to the source in the rest of the energy range denoted as $S_{\text {scat }}^{2}$. The former has positive value over energy while the latter oscillates with neutron energy as displayed in Figure 3. Calculations were then performed for the noise induced by the two source components separately and compared with the total noise. Figures 15 and 16 display the comparison of the amplitude and the phase of the noise in groups 6 and 20, respectively. It can be seen that the two noise components are out-of-phase. Thus, they cancel together in the total noise. Since the amplitude of the noise of $S_{\text {scat }}^{1}$ is greater than that of $S_{\text {scat }}^{2}$, the total noise has the same phase with that of $S_{\text {scat }}^{1}$. This indicates that the properties of the total noise are dominated by the perturbation in a few fast groups.

Calculation of the detector noise has also been performed with the use of two detector types (fission chambers) consisting of ${ }^{235} \mathrm{U}$ and ${ }^{242} \mathrm{Pu}$ fissionable materials, respectively, as used in [15]. The responses of the two detectors, denoted as $\delta \phi_{\text {det }}$, as functions of detector positions along the core radius were obtained. Figure 17 shows the amplitude and phase of the ${ }^{235} \mathrm{U}$ detector noise with the frequencies of $1 \mathrm{~Hz}$ and $100 \mathrm{~Hz}$. Figure 18 shows the same quantity but with the ${ }^{242} \mathrm{Pu}$ detector. Again, one can see that the phase of the detector noise induced by $S_{\text {abs }}$ and $S_{\text {scat }}$ is approximate and opposite to that induced by $S_{\text {fis }}$. Since the ${ }^{235} \mathrm{U}$ detector is more sensitive to thermal and epithermal neutrons, it recognizes well the noise in the reflector region. The ${ }^{242} \mathrm{Pu}$ detector highlights the noise in the fuel region and the local peak at the source position. 


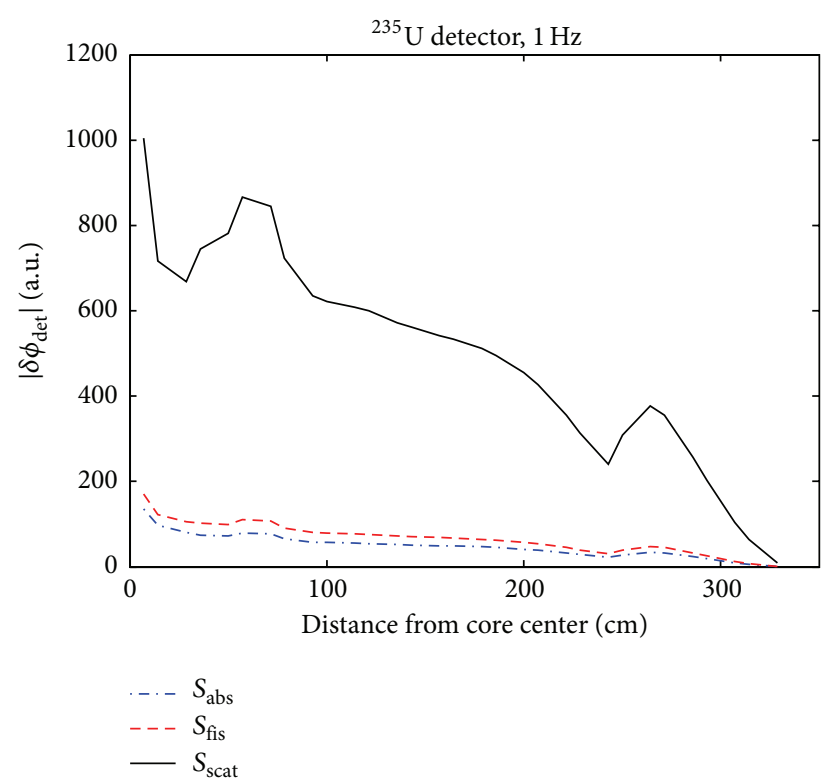

(a)

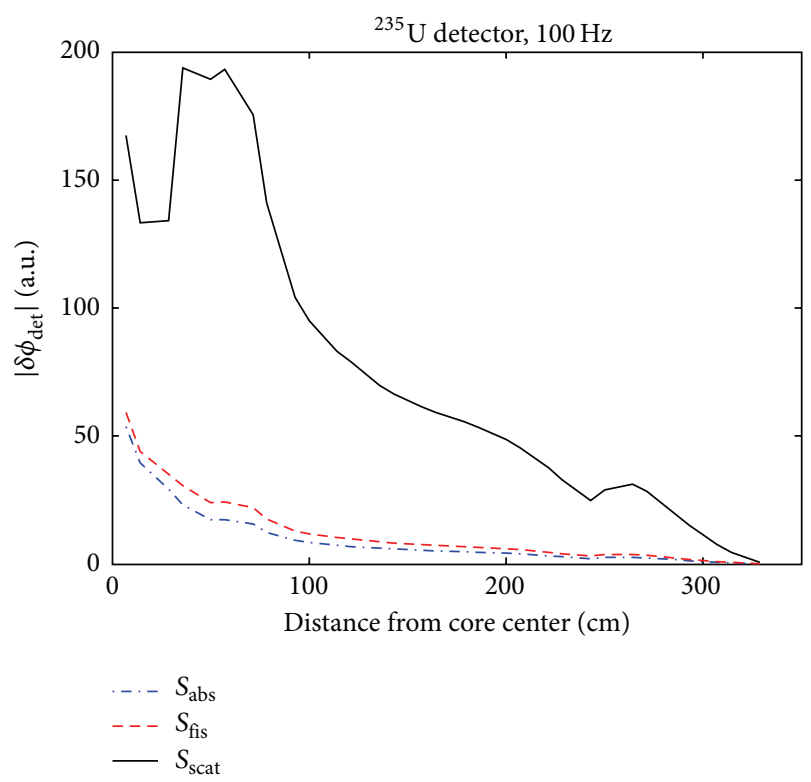

(c)

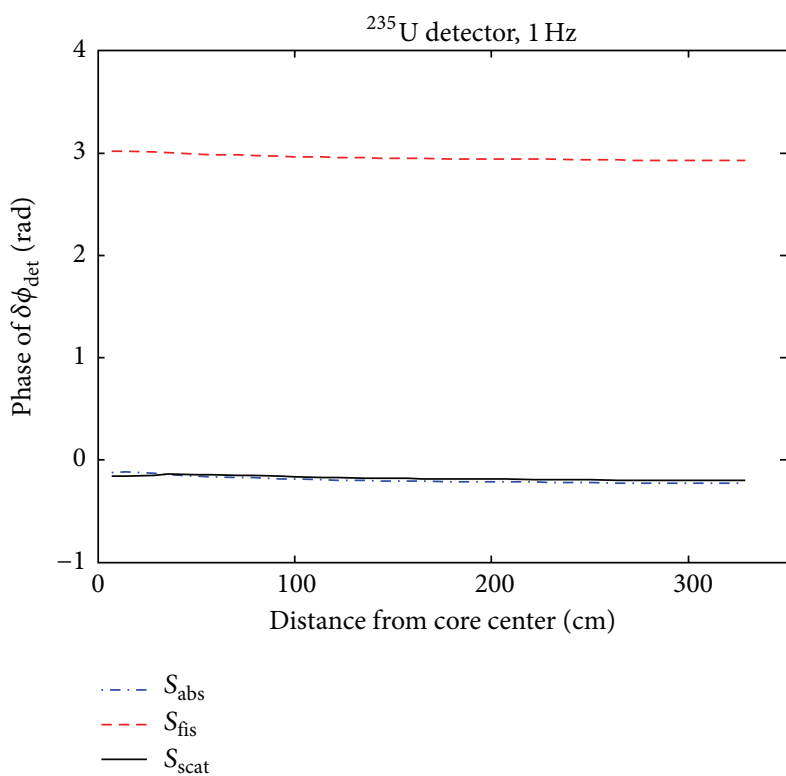

(b)

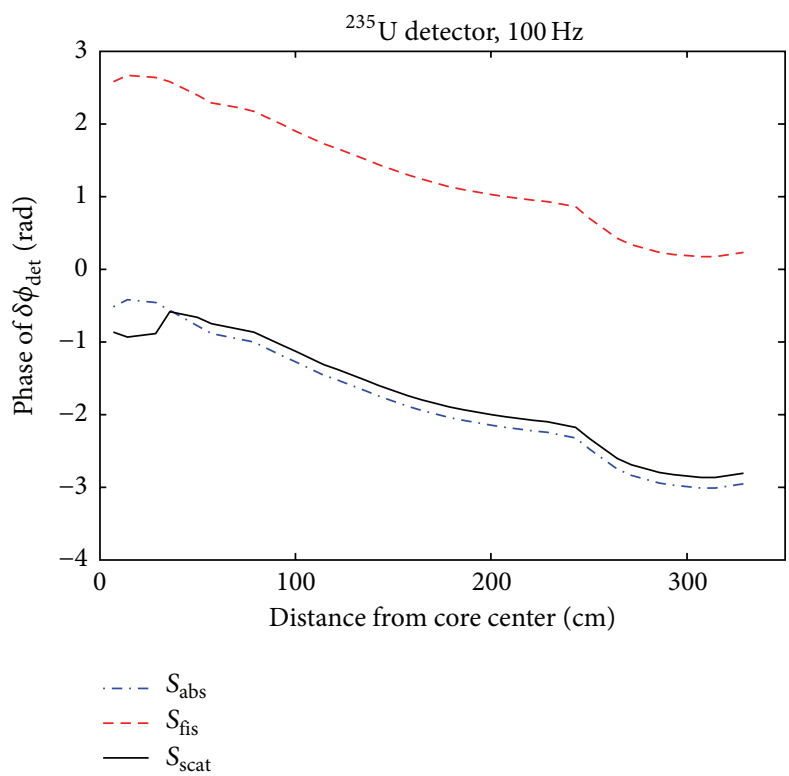

(d)

Figure 17: Amplitude ( $\mathrm{a}$ and $\mathrm{c}$ ) and phase ( $\mathrm{b}$ and $\mathrm{d}$ ) of the neutron noise calculated with the ${ }^{235} \mathrm{U}$ detector at the frequencies of $1 \mathrm{~Hz}(\mathrm{a}$ and b) and $100 \mathrm{~Hz}$ (c and d).

\section{Conclusions}

The properties of the neutron noise induced by localized perturbations in an SFR have been investigated. Three representations of the noise sources were assumed to be located at the core center. Numerical calculations were performed in a wide range of frequencies. Similar to the static flux, the induced noise amplitude is significant at energy $>1 \mathrm{keV}$. In this energy range, the phase of the noise is almost constant with energy despite the source types. This can be explained by the low effect of $\omega / v$ ratio in the high energy range. Variation of the phase decreases with the increase of the distance from the source and is negligibly small at low frequencies. Calculation of the noise induced by the two source components of the perturbation of the scattering cross section shows that the perturbation in several fast groups has a significant contribution and dominates the amplitude and the phase of the induced noise.

\section{Conflict of Interests}

The author declares that there is no conflict of interests regarding the publication of this paper. 


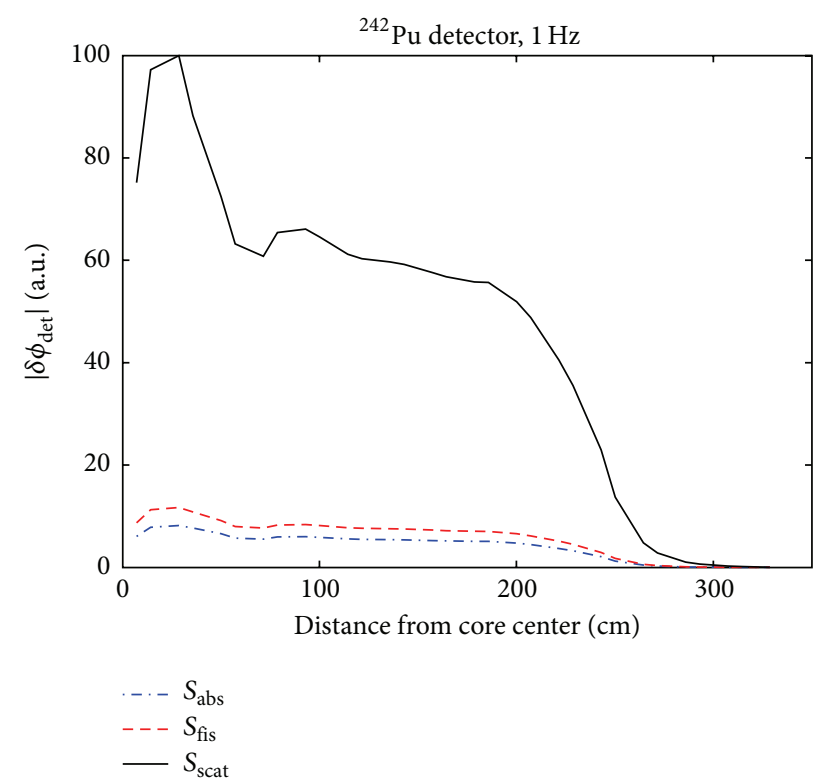

(a)

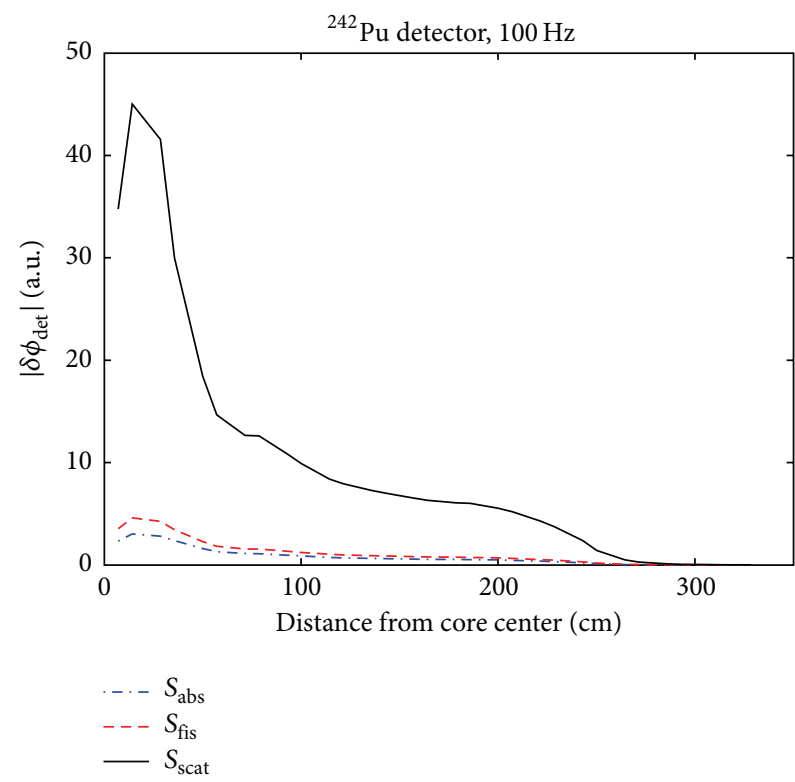

(c)

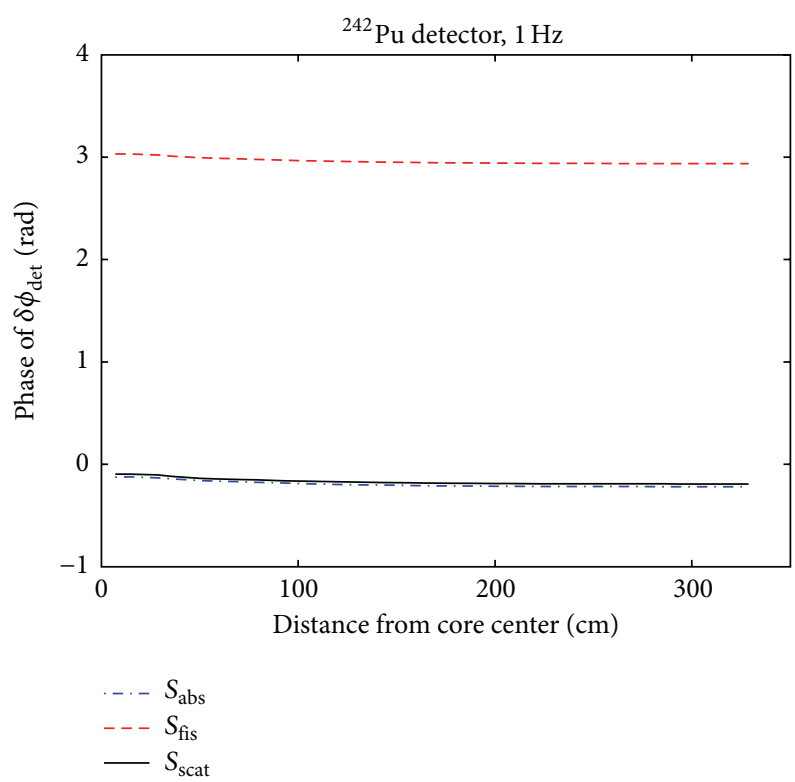

(b)

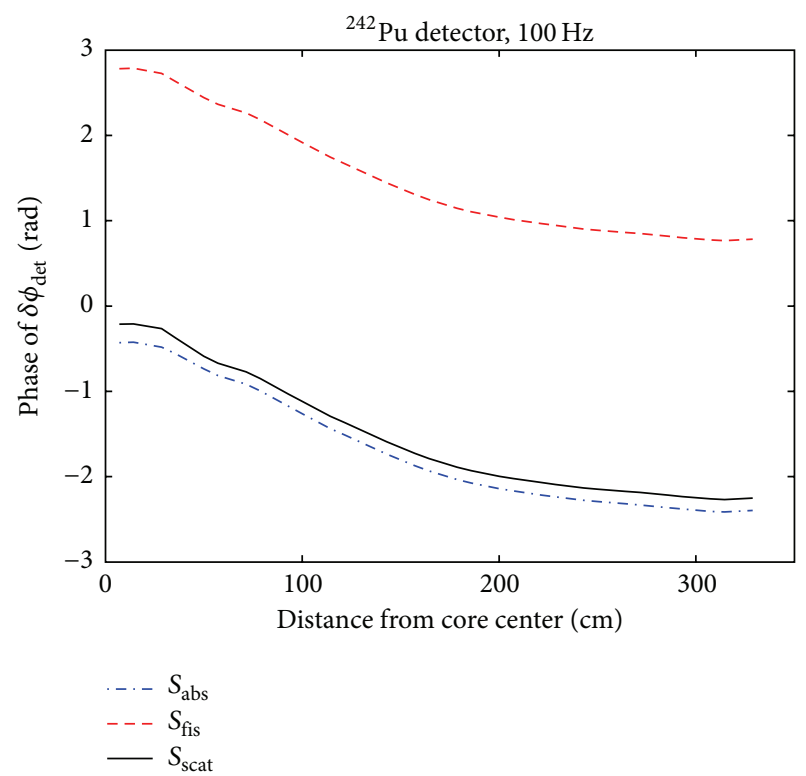

(d)

Figure 18: Amplitude ( $\mathrm{a}$ and $\mathrm{c}$ ) and phase $\left(\mathrm{b}\right.$ and $\mathrm{d}$ ) of the neutron noise calculated with the ${ }^{242} \mathrm{Pu}$ detector at the frequencies of $1 \mathrm{~Hz}(\mathrm{a}$ and b) and $100 \mathrm{~Hz}$ (c and d).

\section{Acknowledgments}

A part of calculations in this work was performed during the author's stay at Chalmers University of Technology. This research is funded by Vietnam National Foundation for Science and Technology Development (NAFOSTED) under Grant no. 103.04-2014.79.

\section{References}

[1] J. A. Thie, "Core motion monitoring," Nuclear Technology, vol. 45, pp. 5-45, 1979.
[2] H. M. Hashemian, "On-line monitoring applications in nuclear power plants," Progress in Nuclear Energy, vol. 53, no. 2, pp. 167181, 2011.

[3] J. Gourdon and R. Casejuane, "Off-line and on-line noise analysis for core surveillance in French LMFBR 'ANABEL", Progress in Nuclear Energy, vol. 9, pp. 365-373, 1982.

[4] T. Umeda, K. Chiba, S. Ebata, Y. Ando, and H. Sakamoto, "Experience of on-line surveillance at onagawa-1 bwr plant," Progress in Nuclear Energy, vol. 21, pp. 35-41, 1988.

[5] D. Wach, "Vibration, neutron noise and acoustic monitoring in German LWRs," Nuclear Engineering and Design, vol. 129, no. 2, pp. 129-150, 1991. 
[6] J. A. Thie, "Fast flux test facility noise data management," Progress in Nuclear Energy, vol. 21, pp. 173-180, 1988.

[7] G. le Guillou, R. Berger, and M. Brunet, "Boiling detection in fast reactors by noise analysis studies performed in France," Progress in Nuclear Energy, vol. 1, no. 2-4, pp. 409-426, 1977.

[8] C. Demaziére, "CORE SIM: a multi-purpose neutronic tool for research and education," Annals of Nuclear Energy, vol. 38, no. 12, pp. 2698-2718, 2011.

[9] V. Larsson, C. Demaziére, I. Pázsit, and H. N. Tran, "Neutron noise calculations using the analytical nodal method and comparisons with analytical solutions," Annals of Nuclear Energy, vol. 38, no. 4, pp. 808-816, 2011.

[10] V. Larsson and C. Demaziére, "A coupled neutronics/thermalhydraulics tool for calculating fluctuations in Pressurized Water Reactors," Annals of Nuclear Energy, vol. 43, pp. 68-76, 2012.

[11] S. A. Hosseini and N. Vosoughi, "Neutron noise simulation by GFEM and unstructured triangle elements," Nuclear Engineering and Design, vol. 253, pp. 238-258, 2012.

[12] P. Filliatre, C. Jammes, B. Geslot, and L. Buiron, "In vessel neutron instrumentation for sodium-cooled fast reactors: type, lifetime and location," Annals of Nuclear Energy, vol. 37, no. 11, pp. 1435-1442, 2010.

[13] H. N. Tran and C. Demazière, "Neutron noise calculations in hexagonal geometry and comparison with analytical solutions," Nuclear Science and Engineering, vol. 175, no. 3, pp. 340-351, 2013.

[14] H. N. Tran, F. Zylbersztejn, C. Demaziére, C. Jammes, and P. Filliatre, "A multi-group neutron noise simulator for fast reactors," Annals of Nuclear Energy, vol. 62, pp. 158-169, 2013.

[15] F. Zylbersztejn, H. N. Tran, I. Pázsit, P. Filliatre, and C. Jammes, "Calculation of the neutron noise induced by periodic deformations of a large sodium-cooled fast reactor core," Nuclear Science and Engineering, vol. 177, no. 2, pp. 203-218, 2014.

[16] G. L. Fiorini and A. Vasile, "European commission-7th framework programme: the collaborative project on European sodium fast reactor (CP ESFR)," Nuclear Engineering and Design, vol. 241, no. 9, pp. 3461-3469, 2011. 


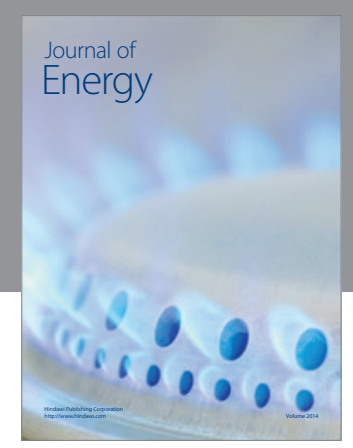

Journal of

Industrial Engineering
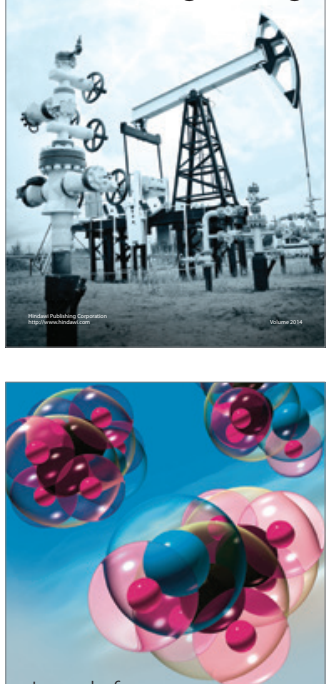

Fuels
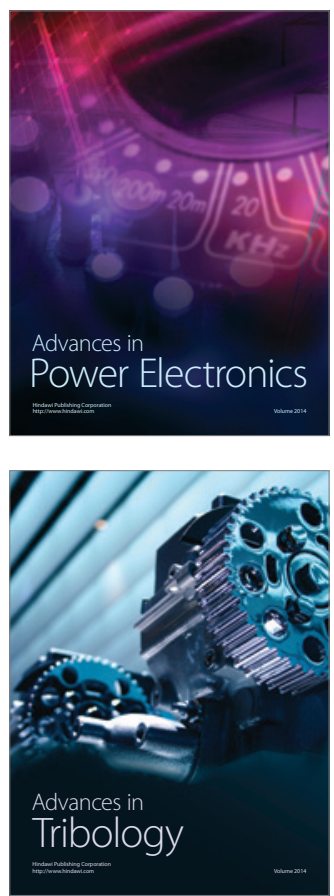

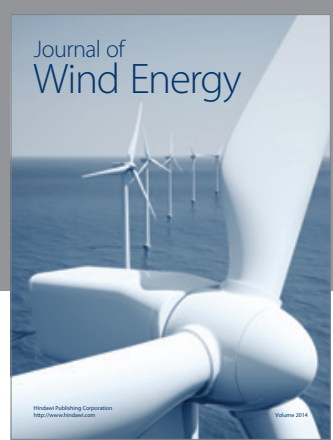

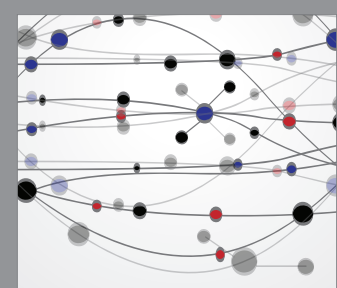

The Scientific World Journal

Submit your manuscripts at http://www.hindawi.com

Journal of

Structures
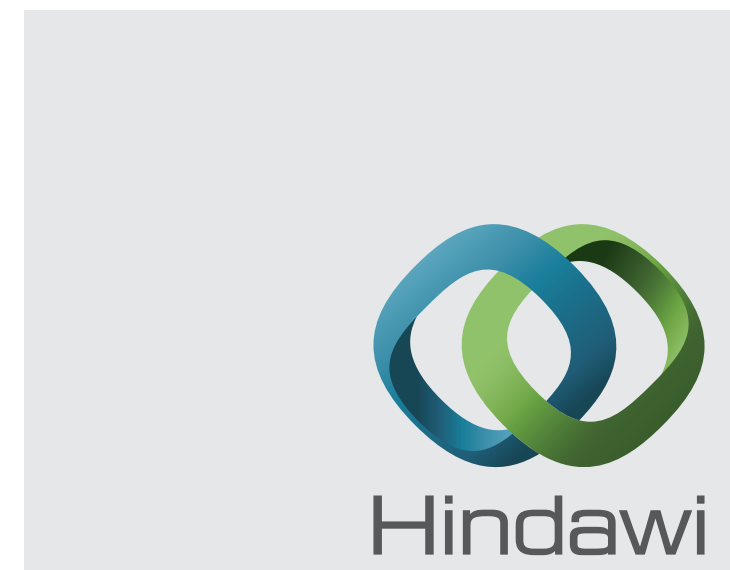

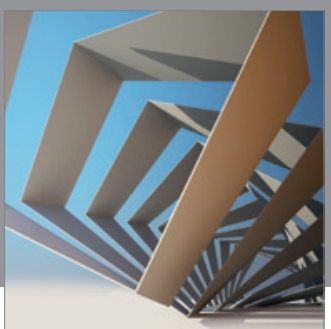

Rotating

Machinery
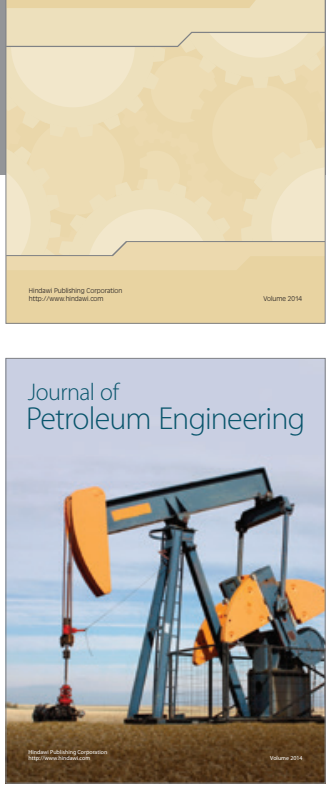

Journal of

Solar Energy
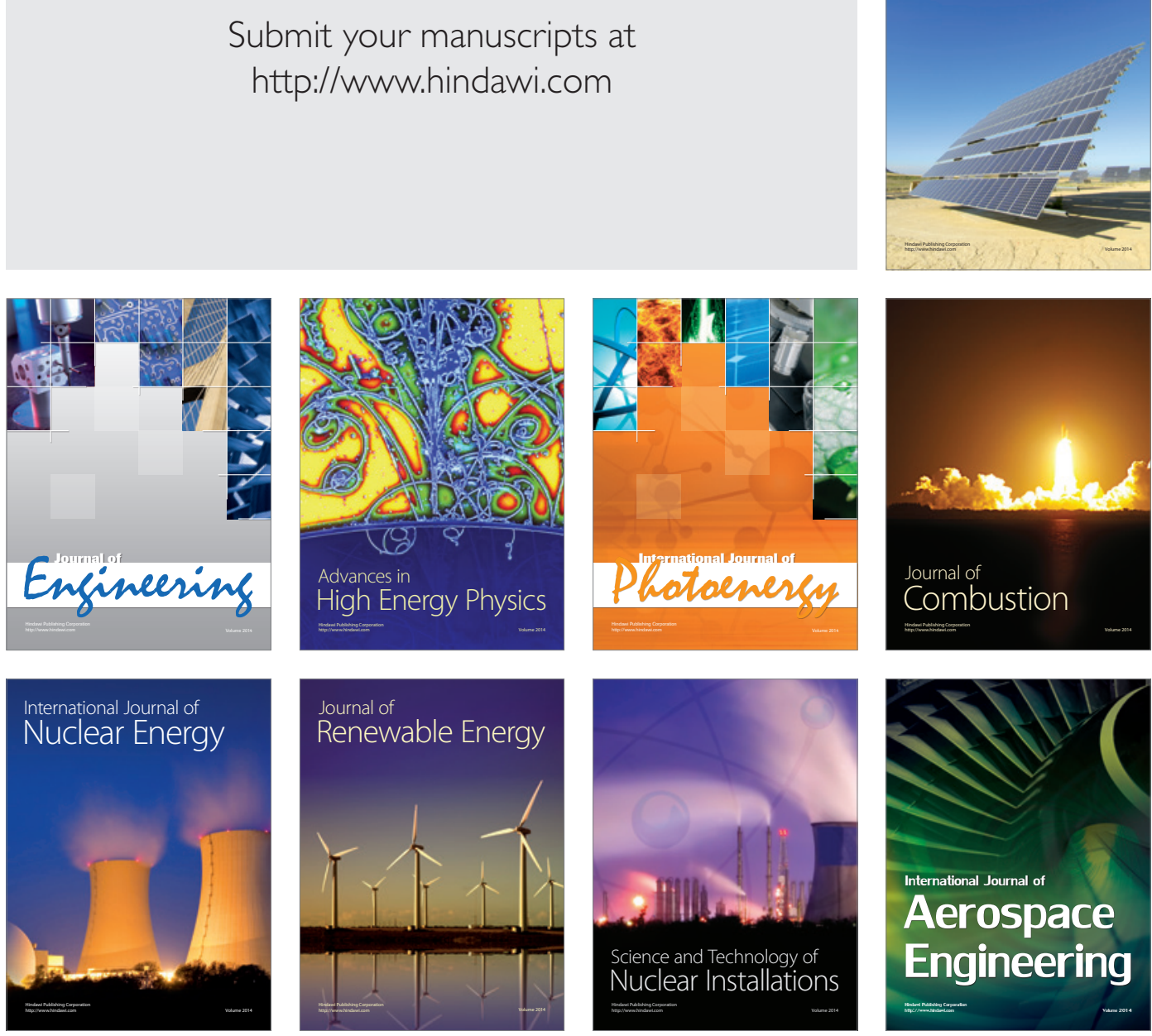